\title{
日本動物心理学会第76回大会発表要旨
}

発表要旨本文は発表者から送られてきた原稿をそのまま記載した。 



\section{Abstracts of Oral Presentations}

O-23-01

Can multiple-item array task test same/different concept in budgerigars (Melopsittacus undulatus) and pigeons (Columba livia)?

Sota Watanabe ${ }^{1)}$, Masayuki Yamada ${ }^{1)}$, Yuriko Hase ${ }^{1)}$, KaZUO Fujita ${ }^{2)}$, MASATO ISHIDA ${ }^{1)}$

${ }^{1)}$ Osaka Kyoiku University, ${ }^{2)}$ Graduate School of Letters, Kyoto University

To investigate the tendency of same-different conceptualization in non-human animals, Wasserman and his colleagues devised and have used multiple-item array task. In this task, subjects are displayed two choice-keys and an array of multiple (typically 16) icons either in a uniform manner (1 pattern) or all to be different (16 patterns). Subjects are required to select the correct choice-key depending on the array. Wasserman \& Young (2010) argued that pigeons and baboons have same/different concept but the concept is controlled by entropy. In this study, we used four budgerigars (Melopsittacus undulatus) and three pigeons (Columba livia), and conducted the same experiments at first as their studies and had the same results as theirs. Next, we conducted the same task except using the arrays those entropy value was controlled. Both of two species showed discriminative response according to the number of icons. These results suggest the two species solved the multiple-item task depending on neither entropy nor same/different concept.

O-23-02

You look/smell familiar: individual difference in sensory modalities used for individual recognition in medaka

MU.YUn WANG ${ }^{1)}$, HideAKI TAKEUCHI ${ }^{2)}$

${ }^{1)}$ Graduate School of Arts and Sciences, The University of Tokyo,

${ }^{2)}$ The Graduate School of Natural Sciences and Technology, Okayama University

Individual recognition (IR) is the essential capability for maintaining various social interaction in animals. Looking at which cue animals use to perceive particular conspecifics can shed light on the evolutionary basis of their social world. Medaka females prefer familiar males and decrease the latency to mate after visual familiarization. We therefore tested which cue is used for IR and the cognitive recognition of medaka, using mating test and electric shock two-alternative forced-choice (TAFC) test. We found both visual and olfactory cues were used for mate discrimination, and medaka shows individual difference in modality used for IR. Some females accepted the males faster after visual familiarized with the male, while others were better in odor familiarization test. Visually-oriented females also passed the TAFC test and successfully detect the change of male after visual familiarization. Medaka used only appearance but not motion cue for individual recognition, and signals around the head might be important; however, when we painted the male face with black ink after familiarisation, the female could still recognise the male. When flip the fish horizontal with prism during familiarisation, females recognised him as the same male, while when the image was upsidedown the female failed to recognise the male.

O-23-03

Inequality aversion in mice: comparison of advantageous and disadvantageous conditions SHIGERU WATANABE

Keio University

Inequality aversion was examined in male C57BL6 mice. The body surface temperature was measured by a thermograph as an index of aversion. I compered advantageous and advantageous inequality in two different situations, namely restraint stress and unequal food delivery. Experiment 1: Stress-induce hyperthermia ( $\mathrm{SIH})$ was examined in restrain stress. 
Disadvantageous condition in which one subject was retrained but three cage mates were free resulted in higher SIH than single-stress condition, while advantageous condition in which three cage mates were restrained but one subject was free did not cause SIH. Experiment 2: $\mathrm{SIH}$ was examined in food-deprived mice. Disadvantageous condition in which one subject did not obtain cheese but three cage mates enjoyed cheese resulted in clear SIH, while advantageous condition in which three cage mates did not obtain cheese but one subject enjoyed cheese did not cause SIH. When the subjects received plenty of cheese before experiment, they did not show SIH in the disadvantageous condition. Conclusion: The mice displayed inequality aversion only in disadvantageous inequality. Devaluation, satiated with food before experiment, deduced SIH in disadvantageous inequality of food delivery.

\section{O-23-04}

\section{Effects of pictorial and motion-parallax depth cues on size constancy in pigeons}

YuYa Hataji, KazUo Fujita

Graduate School of Letters, Kyoto University

Several studies have shown that avian species use various visual depth cues. However, it is still unclear how they integrate different cues to reconstruct a single 3D scene. We studied pigeons' (Columba livia) use of pictorial and motion-parallax depth cues, using a dynamic version of the "corridor illusion." Pigeons learned to classify into large or small the size of a white target circle on the corridor background made of grids. There were two conditions; static and dynamic. The static condition was the same as the classical corridor illusion. In the dynamic condition, the target and the corridor moved sideways so that they could add a motion-parallax cue. The motion amplitude was larger for "nearer" objects. In the test that followed, we inserted probe test trials in which target position and motion amplitude changed independently. Results showed that the pigeons perceived a corridor illusion in both conditions and this perceptual bias was induced by the modulation of pictorial cues, not motion-parallax cues. The human experiment using the same stimuli showed similar dominance of pictorial cues. These results suggest that pictorial cues are more reliable than motion-parallax cues for both species and they share a part of size constancy mechanisms.

\section{O-23-05 \\ Do differently colored objects enhance object-based attention in pigeons? \\ KAZUKI FuJII ${ }^{1)}$, YUKiKo Hoshino ${ }^{2)}$, MAKI KATSUBE ${ }^{2)}$, TOMOKAZU USHITANI ${ }^{2)}$ \\ ${ }^{1)}$ Graduate School of Advanced Integration Science, Chiba University, \\ ${ }^{2)}$ Faculty of Letters, Chiba University}

Humans attend not only to cued areas, but also to cued objects (object-based attention). Humans show a shorter reaction time to targets that appear on cued objects (Within condition) known as within-object benefit, which is not the case when targets appear on noncued objects (Between condition). In our previous study, pigeons showed a within-object benefit when we used differently colored rectangles as background objects and required them to discriminate whether the target appeared on the cued object or not. In the present study, a within-object benefit was observed even in the absence of discrimination, which could have been explained by attention to color, rather than to the object on which the cue appeared. Therefore, we used rectangles with a color gradation, such that the cue and the target appeared on different colors in the Within condition, and on the same color in the Between condition. If pigeons attended to the object color, the reaction time in the Between condition would be shorter than that in the Within condition. However, the results indicated no differences between the two conditions. These findings suggest that other features in addition to color, such as contours might be needed for within-object benefit in pigeons. 
O-23-06

Cross-modal representation of owner in cats (Felis catus)

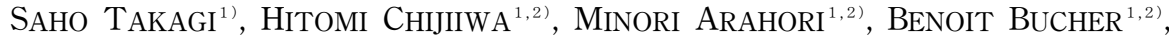
KAZUO FUJITA ${ }^{1)}$

${ }^{1)}$ Graduate school of letter Kyoto University, ${ }^{2)}$ JSPS Research Fellow

We tested whether cats have a cross-modal representation of their owner by an expectancy violation procedure. We presented cats with a photo of either the owner's or a stranger's face on the portable computer after playing back a voice of one of those persons calling the subject's name. The voice and the face matched in half of the trials (Congruent condition) and mismatched in the other half (Incongruent condition). We predicted that cats would look at the monitor longer in Incongruent condition than in Congruent condition, if they have a crossmodal representation of their owner. Cats who kept in cat cafes looked at the monitor longer in both Incongruent conditions as we predicted, whereas cats who kept in a owner's house showed a different looking pattern; they looked at the monitor longer when stranger face appeared after playing back a stranger voice. This results suggests that cats have a capacity to form a cross-modal representation of their owner. However, sensitivity to detect strangers by house cats who do not experience strangers so frequently could overshadow the effect of expectancy violation, possibly because of a characteristics as a territorial species.

O-23-07

Do cats avoid people who behave negatively to their owner?

Hitomi Chijilwa ${ }^{1,2)}$, SAho TAKagi ${ }^{1)}$, Minori ARAhORI ${ }^{1,2)}$, Benoit Bucher ${ }^{1,2)}$, KAZUO FuJITA ${ }^{1)}$

${ }^{1)}$ Graduate School of Letters, Kyoto University, ${ }^{2)}$ JSPS Research Fellow

We showed that dogs avoid people who behaved negatively to their owner (Chijiiwa et al., 2015). In the present study, we tested whether domestic cats similarly evaluate third-party interactions using the same procedure. The owner, after trying to open a lid to take out an object from a transparent container in vain, requested help from an actor sitting next to her/ him. The actor helped the owner to open it in Helper condition $(n=17)$, whereas the actor refused to help by turning away in Non-Helper condition $(n=16)$. A neutral person kept sitting at the other side of the owner in both conditions. After the interaction, the actor and the neutral person both offered a piece of food to the cat who watched the entire interaction. Only one trial was given to each cat. The number of cats who collected food from the actor was 11 (Helper condition) and 7 (Non-Helper condition). Although the difference failed to reach a statistical significance due to small sample sizes, the result appears to suggest that cats discriminate people who behaved cooperatively and non-cooperatively to their owner. We are now testing more cats and will report the final result and discussion at the session.

O-23-08

Rats can reallocate memory resource in a directed forgetting task CHIAKI TANAKA, TOHRU TANIUCHI

Kanazawa University

The present study examined directed forgetting in rats using an eight-arm radial maze. A food cup was set in the middle and at the end of each arm of the maze. A trial consisted of a learning phase, retention interval, and a test phase. In the learning phase, win or lose of a food pellet in the middle cups was signaled whether a large reward would be set in the test phase. Two qualitatively different foods in end cups served as remember- or forget-cues that informed whether those arms would be used or omitted in the test phase. In the test phase, 20 pellets were set in the lose- $\mathrm{R}$ arm and two lose-F arms were excluded from testing by closing doors. After acquisition of the task, probe tests were inserted instead of the normal tests. In the probe test, a lose-F arm was presented as a correct arm instead of a lose- $\mathrm{R}$ arm. 
Rats showed significantly poorer performance in probe trials than in normal trials. The results suggest that rats can selectively control rehearsal in working memory.

\title{
O-23-09
}

Capuchin monkeys (Sapajus apella) know when they should remember: adaptive memory strategies depending upon task difficulty

ReIKI Kishimoto, Sumie IwASAKI, Kazuo Fujita

Graduate school of Letters, Kyoto University

It should be adaptive for animals to change memory strategies based on information value, required cognitive resources and provision for future. Non-human animals have been shown to engage in selective memorization, including ignoring or forgetting useless information and active processing of important information. Therefore, animals should flexibly adjust depth of encoding and amount of effort to maintain the items to be used later in order to maximize benefit at the least cognitive cost. The present experiment provided such an opportunity by informing capuchin monkeys how long the information should be retained at different points; with-sample or after-sample. We thus investigated whether capuchins change informationprocessing depending on their expectation of upcoming delay lengths. Results indicated that two monkeys adopted different strategies depending on whether encoding was controllable but that the third never did. One monkey showed equally deep information encoding regardless of the delay length when encoding was controllable and, in a situation where encoding was uncontrollable, she engaged in active rehearsal especially when her memory trace at the onset of retention was strong enough to last until the offset. The other monkey showed the opposite pattern across conditions. This finding suggests that capuchins know when they should remember or not.

\section{O-23-10}

Do capuchin monkeys have the ability to discriminate age category from conspecific/heterospecific faces?

\author{
YURI KAWAGUCHI ${ }^{1)}$, HikA KUROSHIMA ${ }^{2)}$, KAZUO FUJITA ${ }^{2)}$ \\ ${ }^{1)}$ Primate Research Institute, Kyoto University, \\ ${ }^{2)}$ Graduate School of letters, Kyoto University
}

We can roughly estimate the age of a face of other species as well as that of humans. A possible explanation for that is the "baby schema" (Lorenz, 1943), facial properties commonly observed in babies of many species. However, it is unknown whether animals can also estimate others' age from facial features or whether they have age categories, "adult" and "baby." In study 1, four capuchin monkeys (Cebus apella) were trained to discriminate adult and baby conspecific faces in a symbolic matching-to-sample procedure. Successful transfer of age category to novel stimuli during this training indicated that monkeys have the ability to discriminate age category for conspecifics. Then we tested generalization of this discrimination to $\operatorname{dog}$ and human faces in all-reinforced probe test trials. However, no monkeys succeeded (binominal, two tailed, ps $>0.05$ ). This result suggested that age categorization for own species was limited to their own species. In study 2, we trained naive 2 subjects for age discrimination of human faces. Again they showed transfer of age category to novel stimuli. However, when we tested them with capuchin and dog stimuli in the same procedure, they showed no transfer. These results raise doubts on the concept of generally applicable "baby schema."

O-23-11

Third-party non-reciprocity promotes distrust in tufted capuchin monkeys (Cebus apella)

Benoit Bucher ${ }^{1,2)}$, MARGaux Levasseur ${ }^{1)}$, JAMES ANDERSON ${ }^{1)}$, KAZUO FujiTA ${ }^{1)}$

${ }^{11}$ Department of psychology, Kyoto University, ${ }^{2)}$ JSPS Research Fellow

Previous studies have reported that some non-human animal species can detect reciprocity 
between third parties involved in an exchange of goods. For example, Capuchin monkeys are less willing to accept food from a non-reciprocal human actor than a reciprocal actor. In the present study, we asked whether third-party reciprocity would affect the monkeys' trust toward the actors. After witnessing a reciprocal or non-reciprocal exchange of objects, Capuchin monkeys were tested for delay of gratification as one of the two human actors transferred food, which accumulated within reach of the monkey. In this self-control context, monkeys could grab the food at any moment but this act stopped its accumulation. To maximize the total amount of food obtained, monkeys should therefore restrain themselves as long as possible. Results showed that monkeys were less willing to wait during the accumulation period when the food was transferred by the non-reciprocal actor than by the reciprocal actor. This impulsive tendency was especially observed in a context of uncertainty, i.e. in the condition where the total amount of food available was unknown to the monkeys. These data suggest that non-reciprocity in a third-party exchange can promote distrust in Capuchin monkeys.

O-24-01

The asymmetry defect of hippocampal circuitry impairs working memory in $\beta 2$-microglobulin deficient mice

KAZUHIRO GOTO ${ }^{1)}$, ISAO ITO ${ }^{2)}$

${ }^{11}$ Department of Human Psychology, Sagami Women's University,

${ }^{2)}$ Department of Biology, Kyushu University

Left-right (L-R) asymmetry is a fundamental feature of brain function, but the mechanisms underlying functional asymmetry remain largely unknown. We previously identified structural and functional asymmetries in the circuitry of the mouse hippocampus that result from the asymmetrical distribution of NMDA receptor GluR $\varepsilon 2$ subunits. We found that $\beta 2$-microglobulin $(\beta 2 \mathrm{~m})$-deficient mice that are defective in the stable cell surface expression of major histocompatibility complex class I lack this circuit asymmetry. To investigate the effect of hippocampal asymmetry defect on brain function, we examined the working memory of $\beta 2 \mathrm{~m}$-deficient mice in a delayed nonmatching-to-position task. Mice were trained to nosepoke either a left or right key of a sample, to retain the position of the key during a delay interval, and then to choose the key opposite from the sample. During training sessions, the $\beta 2 \mathrm{~m}$-deficient mice acquired the task as fast as control mice, suggesting that the discrimination of left and right positions is not impaired by the total loss of hippocampal asymmetry. In contrast, the $\beta 2 \mathrm{~m}$-deficient mice made fewer correct responses than control mice when variable delay was imposed, suggesting that the asymmetry of hippocampal circuitry plays an important role in working memory.

O-24-02

Effects of chronic postnatal NMDA receptor blockade on latent inhibition in preadolescent rats Aiman Ismail MuHAmad ${ }^{1)}$, RYo NiIKURA ${ }^{2)}$, KAZUO YAMAdA ${ }^{3)}$, Yukio ICHITANI ${ }^{3)}$

${ }^{1)}$ Graduate School of Comprehensive Human Sciences, University of Tsukuba,

${ }^{2)}$ School of Medicine, Yokohama City University,

${ }^{3}$ Institute of Psychology, University of Tsukuba

Rats treated with N-methyl-D-aspartate (NMDA) receptor, a subtype of glutamate receptor, antagonists in early postnatal period are known to display several behavioral impairments mimicking symptoms of schizophrenia. Niikura et al. (2015) reported that this model of schizophrenia showed deficits in latent inhibition (LI) of conditioned taste aversion (CTA) in adulthood. LI is considered as an ability to ignore irrelevant stimuli, which has been reported to be lacking in schizophrenic patients. In this study, we treated postnatal rats (day 7-20) with an NMDA receptor antagonist, MK-801 $(0.4 \mathrm{mg} / \mathrm{kg})$, twice a day. At the age of 4 weeks, we started water deprivation schedule, and conducted a CTA procedure using sucrose and $\mathrm{LiCl}$. 
Half of the rats were pre-exposed (PE group) to sucrose solution for three days, while the other half were not (NPE group). Chronic postnatal MK-801 treatments did not disrupt the acquisition of CTA itself, and CS-preexposure significantly attenuated subsequent CS-US association (i.e. LI). However, LI deficits induced by postnatal MK-801 treatments did not appear during the developmental stage of preadolescence. Our results suggest the validity of this model by reflecting the onset period of schizophrenic symptoms in human patients.

O-24-03

Why Didn't a Male Long-Tailed Macaque (Macaca fascicularis) Mate in a Harem Cohabitation System?

Maiko KOBAyASHI $^{1)}$, TAKAmasa KOYAma ${ }^{1)}$, YASUHIRO YASUTOMI ${ }^{2)}$, TADASHI SANKAI ${ }^{2)}$

${ }^{1)}$ Japan Women's University,

${ }^{2)}$ National Institutes of Biomedical Innovation, Health and Nutrition

In our previous study, we found $66.7 \%$ of nulliparous females never mated with a male during male-female cohabitation. In this study, we introduced a "harem" cohabitation system of one male and two females to improve mating behavior and social learning. In most cohabitation instances, we did not observe mating. We have analyzed possible reasons why. Among long-tailed macaques bred in the Tsukuba Primate Research Center (TPRC), a fecund male cohabited with nulliparous and multiparous females with regular menstrual cycles; during the study their cages were placed adjacent to each other. Cohabitation of the male/two females was for 7 days, including 11-13 days from onset of menstruation (ovulation period) and their behavior video-recorded. Each morning, the presence of sperm was confirmed via microscope observation of a vaginal smear. When sperm were present, mating was judged successful. In behavioral observation, mating was not successful, although mounting and presenting behaviors were seen. Of the eight females in this ongoing study (four mating instances to date); none have shown sperm in their vaginal smear. We want to learn why and hope to improve mating behavior by changing meal and cohabitation times.

O-24-05

Neural activity in medial frontal areas of human brain for a non-verbal metacognitive task: a preparation for the direct comparison with animal data

SHOKO YUKI ${ }^{1,2)}$, HIRONORI NAKATANI ${ }^{1)}$, TOMOYA NAKAI ${ }^{1,3)}$, KAZUO OKANOYA ${ }^{1)}$, RYOSUKE TACHIBANA ${ }^{1,2,4)}$

${ }^{1)}$ Graduate School of Arts and Sciences, The University of Tokyo,

${ }^{2}$ Japan Society for the Promotion of Science,

${ }^{3)}$ National Rehabilitation Center for Persons with Disabilities,

${ }^{4}$ Institute of Neuroinformatics, University of Zurich/ETH Zurich

Animal studies have explored behavioral evidences of metacognitive process, and recently have begun to investigate its neural bases. Unlike human studies which have directly asked subjects to assess own internal states, studies on animals have indirectly (nonverbally) induced them to make decisions according to their internal states. This methodological discrepancy complicates attempts to explore shared neural mechanisms in humans and animals. To resolve this, we here investigated neural substrate for indirectly induced metacognition in human subjects using a functional magnetic resonance imaging. We measured human brain activations during the delayed matching-to-sample task. Subjects needed to bet either high risk/return or low risk/return options just before the matching according to their memory confidence for maximizing a total score. Brain activations in the dorsal and ventral parts of the medial frontal areas were significantly higher when subjects could perform the bet than when they were forced to choose one of the two options. These regions were also activated in metacognition tasks with verbal self-reference in previous human studies. Results suggest that shared neural substrates exist in verbally and nonverbally investigated metacognitive 
processes. This consistency should facilitate species comparison of metacognition between humans and animals beyond the methodological discrepancy.

O-24-06

Critical evidence for the prediction error theory in the insect learning

Kanta TeraO ${ }^{11}$, Mizunami MaKoto ${ }^{2)}$

${ }^{1)}$ Graduate School of Life Science, Hokkaido University,

${ }^{2)}$ Faculty of Science, Hokkaido University

In associative learning in mammals, it is widely accepted that the discrepancy, or error, between actual and predicted unconditioned stimulus (US) determines whether learning occurs when a stimulus is paired with a US. This theory stems from finding of blocking, but blocking can also be accounted for by other theories. Some behavioral studies to discriminate prediction error theory from alternative theories have performed, but unequivocal evidence to reject all alternative theories has not been obtained in any learning systems. The aims of our study is to find the critical evidence to discriminate prediction error theories from alternative theories. At first, we demonstrated blocking in classical conditioning in crickets. To obtain further evidence supporting the prediction error theory and rejecting alternative theories, we constructed a neural model to match the prediction error theory, by modifying our previous model of learning, and we tested a prediction from the model. Our results suggest that octopaminergic neurons convey reward prediction error in appetitive learning and dopaminergic neurons convey punishment prediction error in aversive learning in crickets. Comparison between insect learning system and mammal dopaminergic system that convey information of reward prediction error would provide further insights for the basic mechanisms of learning and memory.

O-24-08

The role of the insular cortex on underestimation of duration induced by the fear-conditioned tobe-timed stimulus in a temporal bisection task in rats

TAISUKE KAMADA ${ }^{1)}$, TOSHIMICHI HATA ${ }^{2)}$

${ }^{1)}$ Graduate School of Psychology, Doshisha University,

${ }^{2)}$ Faculty of Psychology, Doshisha University

We investigated the role of the insular cortex (IC) in interval timing, time perception in the seconds-to-minutes range, using fear-conditioned to-be-timed stimuli. Rats were first trained on a temporal bisection task in which they have to respond to the lever A after a $2 \mathrm{~s}$ tone, whereas to the lever B after a 8s one. Following training, they were given differential classical conditioning in which one pitch tone $(\mathrm{CS}+)$ was paired with a footshock, whereas the other pitch one $(\mathrm{CS}-)$ was presented alone. They were then infused with either artificial cerebrospinal fluid (aCSF) or $\gamma$-aminobutyric acid A (GABAA) receptor agonist, muscimol, into the bilateral IC before testing in which the bisection task was conducted using the CS+ and $\mathrm{CS}-$ as to-be-timed stimuli. We found that the point of the subjective equality (PSE) in CS+ trials was higher than that in CS- trials with aCSF infusion, while there was no difference between trials with muscimol infusion. These results suggest that the fear-conditioned to-betimed stimulus is judged to last shorter than the neutral one and that the IC involves fear modulation of interval timing.

O-24-09

Effects of the environmental enrichment on the hippocampal volume and spatial information memory in adult mice: longitudinal morphometric study

RiE Ryoke, Akira Sumiyoshi, Hiroi Nonaka, Ryuta KaWAshima

Institute of Development, Aging and Cancer, Tohoku University

Environmental enrichment (EE) is an experimental model which induces neurogenesis and 
dendritic branching in the brain as well as improvements of learning and memory. To understand whether increased brain volumes by $\mathrm{EE}$ would directly relate to learning and memory, we performed the longitudinal study that combined behavioral test and magnetic resonance imaging (MRI) and Golgi-Cox staining in 12 week-old of mice. For the behavioral test, we conducted habituation to open-field for 4 days followed by spontaneous novel object recognition task. For the MRI test, we used voxel-based morphometry (VBM) that can map changes in regional gray matter volume (rGMV) over the time across the entire mouse brain. Both the behavioral and MRI experiments were conducted before and after EE intervention. In open-field task, the habituation index was dramatically changed in the EE mice. The longitudinal VBM analysis showed significant rGMV increases in the dorsal hippocampus of the EE mice. The longitudinal analysis elucidated EE increased hippocampal volumes regardless of inborn volumes. In Golgi-cox staining, the total apical dendritic length in CA1 significantly increased in the EE group. These results suggested that the increment in dorsal hippocampal volume implied the enhancement of visuospatial information memory in $\mathrm{EE}$.

\section{O-25-01}

Should we apply physiological time rather than physical time to an inter-trial interval (ITI)?

\section{MASATO ISHIDA}

Osaka Kyoiku University

Comparative psychologists have sought the optimal experimental conditions for each species, those included apparatus type, quality and quantity of reinforcement, and many other values of independent variables and those of measures and criteria as dependent variables. Time (and frequency), however, has been treated as an absolute scale, and we have never thought time as a relative scale. Some of audience once gave me comments to my presentation which concluded that the length of ITI was one of the important determinants based on the studies on the paradoxical learning effects using rats, turtles, newts and fish. He suggested that a long ITI such as 24-hr was unfair particularly among small-sized animals because of their short life span. The comments might come from physiological time hypothesized and formulated by McMahon (1973). Estimation according to the equation from his theory of elastic similarity illustrates that the $24 \mathrm{hrs}$-ITI for humans will be equivalent to four days for rats and nine days for newts. There are arguments over the meaning of time between animals depending on their biological status (e.g., warm vs. cold blooded). Since physiological time still be a hypothesis (Schmidt-Nielsen, 1984), treating time as an absolute scale would be among our best choices.

\section{O-25-02}

Effect of metronomic sounds on pecking behavior of budgerigars: A study using two response keys

\section{YOSHIMASA SEKI}

Faculty of Letters, Aichi University

Spontaneous music entrainment was reported in 2 parrot individuals (Patel et al., 2009). Then, Hasegawa et al. (2011) reported a rhythmic synchronization in a key peck operant task of budgerigars. Those studies support Rhythmic synchronization and vocal learning hypothesis. The present study attempted to see a spontaneous entrainment to metronomic sounds on a key peck task of budgerigars using an operant conditioning technique. The operant system equipped a left key and a right key and budgerigars were trained to peck an illuminated key. When the left key was illuminated and a bird pecked the key, then the illumination was immediately turned off and illumination of the right key turned on simultaneously, vice versa. A food reward was provided after each 6 pecks. So that, the task made the bird to generate a reciprocal body movement and the peck timing was completely depended on the bird. Then, a metronomic sound was presented in the background throughout in a session that the bird 
was engaging in the task. I observed effects of the rhythm of the metronomic sounds on the rhythm of the key pecking behavior. A variety of inter onset intervals was used for the metronomic sounds.

\section{O-25-03 \\ Perception of subjective contours in pigeons (Columba livia) \\ TOMOKAZU USHITANI, SHIORI MOCHIZUKI \\ Faculty of Letters, Chiba University}

Studies have demonstrated that animals, such as monkeys, cats, fish, and bees perceive subjective contours. However, there has been no firm demonstration of this phenomenon in pigeons based on behavioral measures. One problem in investigating animal perception of subjective contours is that results seemingly suggestive of perceiving subjective contours often cannot exclude the possibility that animals learn to discriminate specific local features formed by the real contours of the inducers. Therefore, the current study was designed to investigate whether pigeons could perceive subjective contours, by using a variety of inducers to inhibit the pigeons from learning to use specific features. More specifically, we trained pigeons to search for an illusory triangle, or square, among illusory squares, or triangles. Both figures were formed on 15 textures consisting of a distribution of many small figures. After the pigeons successfully learned to search for the target, we newly introduced five more textures as inducers (transfer test in Experiment 1) and smaller targets and distractors (Experiment 2). In both tests, the discrimination was successfully transferred to these novel inducers, or items, without any difficulty. This finding strongly supported the perception of subjective contours by pigeons.

\section{O-25-04}

The learning abilities of harbor seal (Phoca vitulina) to novel object and prey

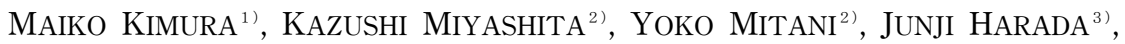
MINA ONODERA ${ }^{3)}$, AYAKO YAMADA ${ }^{3)}$, AI TAJIMA ${ }^{3)}$

${ }^{1)}$ Graduate School of Environmental Science, Hokkaido University,

${ }^{2)}$ The Field Science Center for Northern Biosphere, Hokkaido University,

${ }^{3}$ Asamushi Aquarium

In the eastern Hokkaido, a conflict between harbor seals (Phoca vitulina) and fisheries has become a big problem. Many attempts to scare the seals away from the fishing grounds have been failures in a short time; the seals react to the novel objects or sounds initially but soon get used to and ignore them. It may be caused by great learning ability of seals to understand these novel stimuli are harmless. However, the learning process of seals haven't proved in detail. In this study, we investigated responsiveness to novelty in 4 harbor seals in Asamushi aquarium, Aomori. There were 3 experimental conditions: novel live prey (Plural species), cut of novel prey (Plural species), and control (Daily prey, cut of fish). We presented each prey attached to a novel object (pseudo fishing net) and recorded by $2-3$ video cameras. The analysis by ANOVA suggested that the responsiveness overall varied among individuals; some seals showed positive response toward novel objects, whereas the others showed negative. And seals showed different temporal changes in the agressiveness to the prey, increase or decrease. Furthermore, responsiveness was different by 3 experimental conditions. Our findings revealed that the seals displayed a different cognitive response to prey.

O-25-05

Origin of captive ruffed lemur's reaction to blue color

SANA INOUE

Faculty of Human Sciences, Konan women's University

Red ruffed lemur (Varecia rubra) and black and white ruffed lemur (Varecia variegata) are 
closest species that used to be categorized as sub species. Both species are known as unusual primates with variability in color vision across individuals. However, much of their visual abilities are still unknown. In a previous research, captive lemurs demonstrated lower performance with the blue color stimulus on a match-to-sample task both in terms of accuracy (blue 28\%, red 72\%, yellow 81\%) and reaction time (blue $9516 \mathrm{~ms}$, red $2771 \mathrm{~ms}$, yellow $2761 \mathrm{~ms}$ ) (Inoue and Shindo 2014). The results arouse a question, why lemurs behave differently with blue color? I did a field research with black and white lemur in Torotorofotsy-Ihofa, Madagascar, the lemurs have typical behavior of hang upside down from a branch like bats. In the observation, lemurs frequently showed the behavior to reach a thin branch with fruits against the blue sky, which indicates to ignore the shining blue as a background might be important to ensure smooth foraging. Though further detailed observation would be required, this leads a possibility of understanding the origin of the different response to blue color in captivity.

\section{0-25-06}

Limited spread of experimentally-induced arbitrary tradition in Japanese macaques

Claire WATSON $^{1)}$, NaOKO Hashimoto ${ }^{1)}$, Miya Hamai ${ }^{1}$, Tetsuro MatsuZaWa ${ }^{1,2)}$

${ }^{1)}$ Primate Research Institute, Kyoto University,

${ }^{2}$ Kyoto University Institute for Advanced Study

Communicative and other social traditions represent a large proportion of potential cultural variants observed in nonhuman primates, yet remain understudied experimentally relative to food-oriented traditions. Social behaviors can be relatively arbitrary in form with meaning conferred by shared convention among group members. The ability to learn arbitrary objectrelated action-sequences from others has been demonstrated in captive chimpanzees (Bonnie et al., 2007). Using a somewhat similar paradigm, we investigated whether monkeys (Macaca fuscata) could copy an entirely novel, arbitrary, object-related, action-sequence: picking up and placing a token into one of two differently shaped receptacles, in an open diffusion experiment. Conspecific demonstrators, one from each of two groups $(n=30)$ housed in large outdoor enclosures, were trained alternate target behaviors. Demonstrators then performed the behavior in view of their respective group-mates, across ninety 45-min-per-day sessions. Observers had adequate opportunity to perform the behavior. One juvenile within each group placed tokens in a receptacle. Our results suggest the capacity exists in Japanese macaques, but that spread of such novel arbitrary action-sequences is limited, perhaps partly by functional fixedness. These findings aid understanding of cultural transmission of social traditions in wild monkeys and phylogenetic origins of human social culture. Supported by JSPS.

\section{O-25-07}

\section{Perceptual categorisation of primate faces by chimpanzees}

\section{DUNCAN WILSON, MASAKI TOMONAGA}

Primate Research Institute, Kyoto University

How do chimpanzees categorise primate species based on faces? In a series of touch screen matching-to-sample tasks, six adult chimpanzees were tested on their ability to categorise colour and grayscale identical and same species (non-identical) images of great ape faces (chimpanzees, gorillas and orangutans). In both colour and grayscale conditions, chimpanzees showed good performance for categorising identical faces, but performance decreased for categorising same species faces. Next, the chimpanzees were tested on their ability to categorise grayscale perceptually different primate faces, i.e. humans vs. chimpanzees, which they had previously experienced in real life, and baboons vs. capuchin monkeys, which they had never experienced. They showed relatively good performance for categorising both identical and same species human and chimpanzee faces, and capuchin monkey and baboon 
faces. However, performance decreased for categorising perceptually similar same species gorilla and orangutan faces, which they had also never experienced. The results suggest perceptual similarity may be more important than low-level perceptual cues (e.g. colour) or previous experience for categorising primate faces. Overall, perceptual similarity, low-level perceptual cues, and long-term experience likely interact to influence perceptual categorisation of primate species based on faces.

\section{O-25-08}

\section{Attention to inattention: Perception of the other's gaze by chimpanzees}

MASAKI TOMONAGA ${ }^{1)}$, TOMOKO IMURA ${ }^{2)}$

${ }^{1)}$ Primate Research Institute, Kyoto University,

${ }^{2)}$ Niigata University of International and Information Studies

Faces include important social information such as gaze. We already reported the chimpanzee's sensitivity to dyadic gaze (direct vs. averted gaze) under the visual search task. And they can follow the other's gaze direction which is basis for joint attention or triadic gaze interactions. It is, however, still unclear how they perceive the other's gaze interactions. In the present study we tested the visual search performances using the various photographs in which human model holding an object with or without paying attention to it. Chimpanzees were required to touch the specific target object irrespective of the attentional state of the model. As a result, they showed quicker detection when the target object was not attended by the model than vice versa. This finding was consistent with the results from human infants. In addition, this finding was replicated by using the photographs with the chimpanzee model. Furthermore, when the (human or chimpanzee) model was masked with black color (silhouette condition), this effect was not observed, ruling out the possibility of the effect of configuration. For chimpanzees, the other's inattentional state more readily captures their attention.

\section{Abstracts of Poster Presentations}

\section{P-24-01}

Perception of figure element size by pigeons is affected by element arrangements

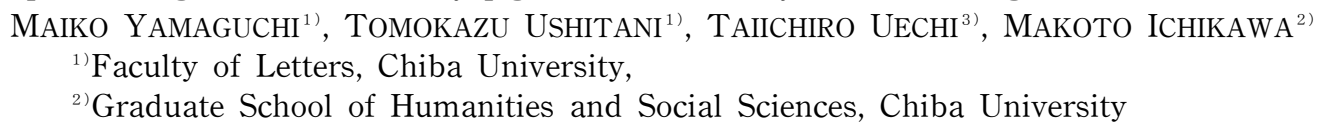

Uechi and Ichikawa (2016) reported an optical illusion in which the size of figure element disks forming a circular array was perceived as smaller when the array was larger in diameter (i.e., the disks were sparsely arranged). Unlike Ebbinghaus illusion, this new illusion may be caused by size contrast between global configuration and local elements rather than between local elements. We examined if pigeons would perceive this illusion by first training four pigeons to discriminate the size of a singly-presented disk. Specifically, a single disk appeared at the display center and pecking at it produced comparisons. When the diameter of the disk was 8,10 , or $12(20,22$, or 24$)$ pixels, the pigeons were rewarded by choosing the comparison corresponding to small (large) disks. After the pigeons had learned this task, we tested the pigeons with multiple disks of 14,16 , or 18 pixels in diameter that were circularly arranged on non-reward probe trials. The diameter of the circular array was either 60, 100, or 140 pixels. Results showed that larger was the diameter of the circular arrangement, more frequently did the pigeons select the "large" comparison. These results suggest that pigeons perceive this illusion similarly as do humans. 


\section{P-24-02 \\ Dog Breed differences of the Oxytocin Receptor Gene \\ YUMA NAITO ${ }^{1)}$, AKITSUGU KONNO ${ }^{1)}$, MiHO InOUE-MURAYAMA ${ }^{2)}$ \\ ${ }^{11}$ Department of Animal Sciences, Teikyo University of Science, \\ ${ }^{2)}$ Wildlife Research Center, Kyoto University}

In this study, we investigated genetic diversity of the oxytocin receptor gene (OXTR) in dogs (Canis familiaris). The rs8679682 SNP in exon 3 of the OXTR was genotyped with nine European breeds (e.g., Labrador Retriever, German shepherd) and 11 breeds native to non-European countries (e.g., Siberian Husky, Akita). The rs8679682 SNP possessed two different alleles (T or C), and both of the resulting codons (GTT, GTC) encoded valines (i.e. synonymous variant). Results showed that allele frequency of the SNP significantly differed according to dog breeds, for instance, with that the $\mathrm{T}$ allele was common in Labrador Retriever whereas the $\mathrm{C}$ allele was common in German shepherd. We will discuss a role for the OXTR variants in evolutionary process of dog breeds by comparing geographical origin, purposes of breed creation, and genetic differences among dog breeds.

\section{$\mathrm{P}-24-03$}

Oxytocin may enhance "attractiveness" of female mice

TOMOYA SugAnUMA, KOHJI TAKADA

Department of Psychology, Teikyo University

Rewarding effects of oxytocin $0.1 \mathrm{mg} / \mathrm{kg}$, s.c., were examined using the conditioned place preference (CPP) paradigm in female $\mathrm{C} 57 \mathrm{BL} / 6 \mathrm{~J}$ mice which were housed either individually (Gr. 1) or in groups (4-5 mice per cage; Gr. 2). After a 2-day measure of preference for each compartment, subjects of Gr. 1 were individually conditioned in one compartment after oxytocin injection, and the other after saline injection. This conditioning alternated daily for 3 injections of each. On the test (Day 9), subjects were allowed to explore the compartments freely with dwell time in each compartment measured. Subjects of Gr. 2 were conditioned in the same manner but were confined in a compartment with the same female cage mate, which was not injected. These subjects and their conditioning mates were tested individually on Day 9 as described for Gr. 1. Neither Gr. 1 nor 2 subjects showed a compartment preference. However, the conditioning mates for Gr. 2 subjects showed significant preference for the compartment paired with oxytocin. These results sharply contrast with a previous study in which individually housed males showed aversion to the oxytocin-injected compartment, which was diminished slightly by cage mate pairing.

\section{$\mathrm{P}-24-04$}

Effects of hippocampal AP5 treatment on relearning of radial maze task in rats

SAKI TOUME ${ }^{1)}$, TAICHI ISHII ${ }^{1)}$, MANAMI SUGITA ${ }^{2)}$, YUKIO ICHITANI ${ }^{2)}$, KAZUO YAMADA ${ }^{2)}$

${ }^{1}$ School of Human Sciences, University of Tsukuba,

${ }^{2)}$ Faculty of Human Sciences, University of Tsukuba

Hippocampal N-methyl-D-aspartate (NMDA) receptors, a subclass of glutamate receptors, are commonly believed to be involved in spatial learning and memory. However, studies showed rats treated with NMDA receptor antagonists were able to normally perform spatial memory tasks under a familiar context, but not under an unfamiliar one, suggesting NMDA receptors are not absolutely essential for spatial memory. In this study, we investigated the effects of hippocampal treatment of AP5, an NMDA receptor antagonist, on performance of the radial maze task under both familiar and unfamiliar contexts. Male WIAR rats were first trained in a three-baited/five-unbaited version of the eight-arm radial maze task in a specific context. After the rats reached a learning criterion, their performance was tested under the treatment of the drug or vehicle into the dorsal hippocampus through the bilaterally implanted guide cannulae. Then, the rats were trained the radial maze task, in which different three arms were 
baited in a different apparatus and room (context). After reaching the criterion, the rats received the drug test again. Results showed AP5 impaired reference memory only in the original context but not in the second one, suggesting relearning of radial maze task does not depend on hippocampal NMDA receptors.

\section{$\mathrm{P}-24-05$}

Strategies for jamming avoidance of bats reflect different behavioral contexts KAZUMA HASE $^{1)}$, TAKAFUMI FURUYAMA ${ }^{1,2)}$, YUKIMI KADOYA ${ }^{1)}$, KOHTA I. KOBAYASI ${ }^{1)}$, SHIZUKO HIRYU ${ }^{1,3)}$

${ }^{1)}$ Graduate School of Life and Medical Sciences, Doshisha University,

${ }^{2)}$ JSPS Research Fellow, ${ }^{3)}$ JST, PRESTO

Echolocating bats emit ultrasound pulses and analyze returning echoes for spatial orientation and navigation. Echolocation is often interfered from sounds of conspecifics. However, how bats extract necessary information from background noises remains unknown. Here, we investigated how bats adjust the acoustic characteristics of their emission responding to acoustic interference during crawl in a closed space. We trained two Japanese house bats (Pipistrellus abramus) in a two-alternative forced-choice procedure to discriminate a difference in target distance, by crawling to either arm of a Y-shaped platform, corresponding to the nearer target. The trained bats performing the task were exposed to jamming stimuli mimicking bat pulses. As a result, the bats lengthened their interpulse interval (IPI) under acoustical interference compared to no jamming condition. These results indicate that crawling bats avoid temporal overlaps between their echoes and the stimuli by reducing the number of emission. In contrast, our previous study demonstrated that the bats during flight shifted frequency of emitted pulses to avoid spectral jamming without changing IPI. Taken together, the bats could employ different strategies to avoid jamming according to different behavioral contexts: flying in an open space or crawling in a closed nest.

\section{$\mathrm{P}-24-06$}

Identification of social cues in emotional contagion of pain using MSM/Ms and C57BL/6 strains of mice

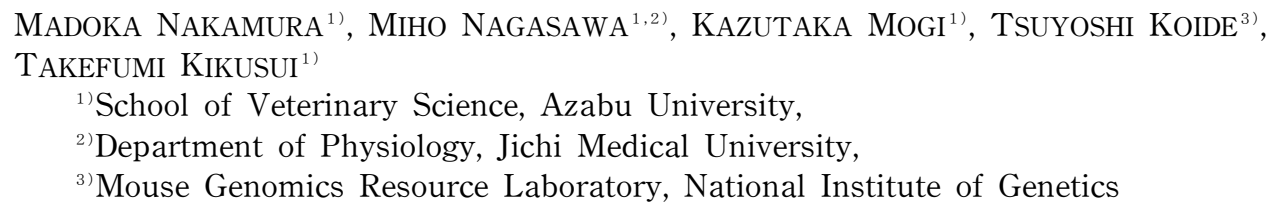

${ }^{1)}$ School of Veterinary Science, Azabu University,

${ }^{2)}$ Department of Physiology, Jichi Medical University,

${ }^{3)}$ Mouse Genomics Resource Laboratory, National Institute of Genetics

Emotional contagion is one fundamental component of empathy that allows individuals to detect the emotional information transmitted by others and express the same emotion. Although recent findings endorse that empathy occurs in many animals including rodents, it is still unknown what specific cues are applied to form the basal layer of empathy. We aimed to determine how mice detect another individual's emotional state without distinguishing the origin of the emotion. The Japanese wild-derived strain MSM/Ms (MSM) mice were tested as observers since previous studies showed that MSM mice are more sensitive to fear-eliciting situations as compared to the standard laboratory strain C57BL/6 (B6). Observers were shown footage on an iPad mini tablet of unfamiliar B6 mice receiving painful foot shocks. At least one of the following stimuli was presented during observation: (a) urine collected from a shocked B6 mouse, (b) urine collected from a non-shocked B6 mouse or (c) distilled water. Consequently, the MSM observers under condition (a) showed significantly higher freezing behavior than the other two conditions. These results suggest a strong possibility that olfactory information is one of the effective social cues in emotional contagion of pain in mice. 
$\mathrm{P}-24-07$

Effects of blood pressure reduction on working memory task performance in spontaneously hypertensive rat: A relationship between dosage of hydralazine and the retention gradient

TOSHIHIKO SATO

Graduate School of Health and Environmental Sciences, Tohoku Bunka Gakuen University

This study aimed to determine the effects of blood pressure reduction on memory function in rats. We compared the number of correct responses in a delayed-matching-to-place (DMTP) task in spontaneously hypertensive rats (SHR) and Wistar-Kyoto strain (WKY) rats. After pretraining, rats were tested with the DMTP task four times, and in each test, they were injected with a vasodilator hydralazine at one of four different doses $(0,0.1,0.3$, and $0.6 \mathrm{mg} / \mathrm{kg}$ ) in a randomized order. To obtain a retention gradient, the interval between the presentation of a lever as a sample and that of two levers for choice response was varied among five different durations $(0,2,5,10$, and $20 \mathrm{~s})$ in random order. A three-factor ANOVA and its subsequent analyses indicated that when the delay was $2 \mathrm{~s}$ or more, the number of correct responses gradually decreased proportionally with the length of the delay. Furthermore, the number of correct responses after injecting 0.3 and $0.6 \mathrm{mg} / \mathrm{kg}$ hydralazine was smaller than that after vehicle (saline) injection. However, the differences in memory function between these doses (vehicle and 0.3 or $0.6 \mathrm{mg} / \mathrm{kg}$ ) was relatively small. In conclusion, there was no significant strain difference between SHR and WKY rats.

\section{$\mathrm{P}-24-08$}

Attention enhances auditory peripheral sensitivity for listening weak communication sounds in Mongolian gerbil

YuUta Harada, Hiroyuki Miyawaki, Shizuko Hiryu, Kota Kobayashi

Graduate School of Life and Medical Sciences, Doshisha University

Mongolian gerbils (Meriones unguiculatus) communicate with other individuals by using frequency moderated sound beyond $20 \mathrm{kHz}$. However their auditory sensitivity for that frequency range is relatively high. Therefore we assume that when gerbil listen significant sound, their auditory periphery might amplify a received signal. Amplitude of cochlear microphonic $(\mathrm{CM})$, which is total of receptor potential of auditory periphery, was measured as an index of auditory peripheral sensitivity. Communication sound and the tone burst with no frequency moderation were presented to the gerbil. The $\mathrm{CM}$ response were measured while the subject was placed with and without an anesthetized cage mate for evaluating the effect of behavioral context on $\mathrm{CM}$. We minimize variation of stimulus amplitude introduced by fluctuations in distance between ears and a sound source using earphones system developed for this experiment. As a rest, it was confirmed that $\mathrm{CM}$ amplitude were enhanced when cage mate present. The result suggests that the cochlear amplification could increase auditory sensitivity when animal pays attention the communication sound.

\section{$\mathrm{P}-24-09$}

Effects of thyroid hormone on the synaptic transmission in the pallium of domestic chicks YURIKO SAHEKI $^{1)}$, SHInJI YAMAGUCHI ${ }^{2)}$, KOICHI HOMma ${ }^{2)}$, TOSHIYA MATSUSHIMA ${ }^{3)}$

${ }^{1)}$ Graduate School of Life Science, Hokkaido University,

${ }^{2)}$ Faculty of Pharmaceutical Sciences, Teikyo University,

${ }^{3)}$ Faculty of Science, Hokkaido University

Imprinting is a case of early learning in birds, in which newly-hatched chicks learn to follow conspicuous moving object that they are exposed to. Intermediate medial mesopallium (IMM) of telencephalon plays a critical role in the acquisition of the imprinting memory (Horn 2004). It was recently found that imprinting increases the amount of 3,5,3'-triiodethyronine (T3) in the brain tissue including IMM. Furthermore, intravenous injection of T3 facilitates imprinting 
(Yamaguchi et al. 2012). In this study, we examined how T3 change the synaptic transmission in IMM by using in vitro slice preparations of IMM. We found that bath applied T3 $(40 \mu \mathrm{M})$ acutely enhanced a component of field potential responses, that is referred to as $\mathrm{P}$ wave (a positive wave collectively representing post-synaptic responses). On the other hand, no effect was found in the pre-synaptic responses. The acute T3 effect survived after bath-application of $40 \mu \mathrm{M}$ DNQX (AMPA/kinate type glutamate receptor antagonist). On the other hand, a further addition of $10 \mu \mathrm{M}$ bicuculline (GABA-A type receptor antagonist) weakened the T3 effect. Furthermore, T3 did not change the waveform of bicuculline-induced epileptic discharges, which represent glutamatergic excitation. T3 may disinhibit IMM neurons via suppression of GABA-A-ergic inhibitory transmission.

\section{P-24-10}

\section{Striatal and Tegmental Neurons Code Critical Signals for Temporal-Difference Learning in Domestic Chicks \\ Chentao WeN $^{1)}$, Yukiko OgURA ${ }^{2)}$, Toshiya Matsushima ${ }^{3)}$ \\ ${ }^{1)}$ Graduate School of Life Science, Hokkaido University, \\ ${ }^{2}$ School of Medicine, Hokkaido University, ${ }^{3}$ Faculty of Science, Hokkaido University}

To ensure survival, animals must update the internal representations of their environment in a trial-and-error fashion. Psychological and neurophysiological studies have suggested the basal ganglia are involved in this updating using the temporal-difference (TD) method. However, how the TD method is implemented at the neuronal level is still unclear. We trained domestic chicks to associate color cues with food rewards. After training, neuronal activities from the medial striatum and tegmentum were recorded in a task when the predicted reward was omitted. To compare neuronal activities with TD-learning theory, we simulated three signals: the prediction signal, the target signal for updating, and the TD-error signal, in a similar task. We found two types of striatal neurons successfully mimicked the target signal and the prediction signal. A linear summation of these neurons was a good fit for the activity of one type of tegmental neurons mimicking the TD-error signal. The present study thus demonstrates that the striatum and tegmentum can convey the signals critically required for the TD method. Based on the theoretical and neurophysiological studies, together with tracttracing data, we propose a novel model to explain how the convergence of striatal signals could lead to the TD error in tegmental neurons.

\section{$\mathrm{P}-24-11$}

Selective contribution of the telencephalic arcopallium to the social facilitation of foraging efforts in the domestic chick

QIUHONG XIN ${ }^{1)}$, YUKIKO OGURA ${ }^{2)}$, TOShiYA MATSUSHIMA ${ }^{3)}$

${ }^{1)}$ Graduate School of Life Science, Hokkaido University,

${ }^{2)}$ Graduate School of Medicine, Hokkaido University,

${ }^{3}$ Faculty of Science, Hokkaido University

To understand the neural basis of socio-economic behaviours in birds, we examined effects of bilateral lesion of arcopallium (Arco) and surrounding nuclei in domestic chicks. We tested foraging effort in an I-shaped maze with two food patches that delivered food in a biased manner according to variable interval schedule. Chicks run back and forth between the patches, and the patch use time matched the respective food delivery rate. In the pair phase, even though actual interference of food did not occur, chicks showed a social facilitation of running effort compared with the single phase. Chicks with lesions in Arco and lateral Arco showed a significant reduction in the social facilitation without effects on the matching. The lesion effects of lateral Arco was particularly selective, as it was not accompanied by changes in the running distance in the single phase. No lesion effects were found by lesions in nidopallium or nucleus taeniae of the amygdala. In accordance, anterograde tracing revealed 
characteristic projections of the lateral Arco. Pathways from the lateral Arco could enable chicks to overcome the extra effort investment of social foraging, suggesting functional and anatomical analogies to the anterior cingulate cortex and basolateral amygdala in mammals.

\section{$\mathrm{P}-24-12$}

Effects of imprinting using point-light animations of biological motion on the visual preference in domestic chicks

Nishi DAISUKE ${ }^{1)}$, TOShiYa MATSUSHIMA ${ }^{2)}$

${ }^{1)}$ Graduate School of Life Science, Hokkaido University,

${ }^{2)}$ Faculty of Science, Hokkaido University

Johansson (1973) created a simplified animation of a walking human by replacing body parts with a set of moving points of light, which gave us a vivid sensation of a walking human. This phenomenon has been referred to as biological motion (BM). Recently, newly hatched domestic chicks are reported to show a preference for BM, and the preference is supposed to be innate (Vallortigara et al. 2005, Miura and Matsushima 2012). The BM preference also facilitates filial imprinting in domestic chicks (Miura and Matsushima 2016). As the first step to understand the brain mechanism, we examined how these results were reliably reproduced. Imprinted chicks showed preference for BM, but they did not show a preference to the color associated with BM. Instead, chicks of all groups showed a preference to yellow. As the second step, we investigated whether the visual preferences for BM and colors could change after selective lesions of the visual pathways. Preliminary experiments however did not reveal any significant effects after the bilateral lesions to visual Wulst. Effects of lesions to entopallium must be studied as the next step.

\section{$\mathrm{P}-24-13$}

Arcopallium and operant peck latency in domestic chicks

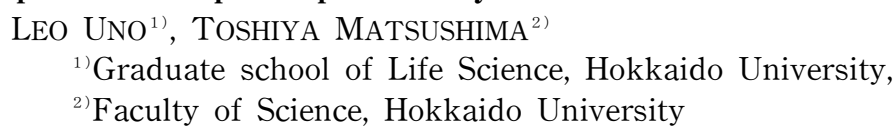

Arcopallium (Arco) is the source of a major descending projections from the avian telencephalon. So far, Arco is ascribed to various functions, such as emotional behavior (Philips 1964), song production (Nottebohm 1976), working memory for sound localization (Knudsen \& Knudsen 1996), and decision making based on handling cost (Aoki et al. 2006). More recently, bilateral lesions of the arcopallium proved to suppress the social facilitation of foraging effort (Xin, Ogura and Matsushima, submitted). Social facilitation has been widely reported as an enhanced frequency or intensity of behavior in the presence of other individuals (Crawford 1939). Therefore, it is possible that Arco is generally responsible for perception of other individuals. If it is the case, Arco is involved also in the response latency that is shortened in competition (Amita and Matsushima 2011). In this study, we examined if the operant peck latency and its social facilitation are suppressed by bilateral lesions of Arco. We did not find significant difference in the latency between intact chicks and those with lesion. The result suggests that Arco is not involved in either perception of other individuals, nor in the behavioral changes that follow. 
$\mathrm{P}-24-14$

Emotional contagion between dogs and their owners

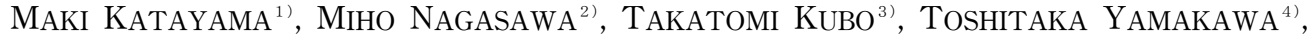

KOICHI FUJIWARA ${ }^{5)}$, KAZUTAKA MOGI ${ }^{1)}$, KAZUSHI IKEDA ${ }^{3)}$, TAKEFUMI KIKUSUI ${ }^{1)}$

${ }^{1)}$ Department of Animal Science and Biotechnology, Azabu University,

${ }^{2}$ Department of Physiology, Jichi Medical University,

${ }^{3}$ Department of Information Science, Nara Institute of Science and Technology,

${ }^{4}$ Priority Organization for Innovation and Excellence, Kumamoto University,

${ }^{5)}$ The Department of Systems Science Graduate School of Informatics, Kyoto University

Emotional contagion is a primitive form of empathy, eliciting a similar emotional state through perceiving anothers' emotional state. Emotional contagion appears to be widespread among individuals within a species. However few studies indicated the presence of emotional contagion between different species. In this study, we investigated whether emotional contagion between dogs and humans exists. We assessed behavioral and physiological parameters (behaviors and heart rate variability) in the stress condition (owners were under social stress) and the control condition (owners have no social stress) for each dog-owner pair and applied StateTrait anxiety inventory to the owners' data before and after the experiment. As a result, the owners' state anxiety rose and the dogs' attention toward their owners increased in the stress condition. These showed when owners felt more stress, dogs observed owners' emotional change. In addition, the HRVs in some dog-owner pairs fluctuated synchronously in both conditions. These suggest that dogs' HRV was changed by perceiving owners' emotional change. We are carrying out an HRV analysis with shorter time-scale to reveal this possibility and discuss whether there is emotional contagion between dogs and owners.

\section{$\mathrm{P}-24-15$}

The desire of pre-weaned calves for stationary cow brush-grooming when maternal grooming is restricted

YUKI KIMURA, DAISUKE KOHARI

United Graduate School of Agricultural Science Tokyo University of Agriculture And Technology

It was reported that cow brush might afford a substitute for maternal grooming. This study was conducted to clarify the desire for brush-grooming when maternal grooming is restricted. 12 Japanese black cattle calves (2-3 months) were used as subject animals. We used a stationary cow brush (B2; DeLaval). Experiments were divided into a control, restricted and released period. In control period, calves could contact their dams without restriction. It was set as the day before the restricted and released periods. In the restricted period, calves were separated from their dams from 10:00 to 13:00. In released period, calves were released and could again contact with their dams from 13:00 to 16:00. We recorded duration of following grooming behaviours using continuous observation methods: self-grooming, grooming using some object except cow brush (object-grooming), social-grooming, maternal grooming and brush-grooming. No differences were observed in self, object and social-grooming in restricted and released periods. However, brush-grooming in the released period was lower than that in restricted period (control $7.8 \pm 12.3 \mathrm{~s} / \mathrm{calf} / \mathrm{h}$; restricted $17.9 \pm 5.7 \mathrm{~s} / \mathrm{calf} / \mathrm{h}$; released $1.5 \pm 0.8 \mathrm{~s} / \mathrm{calf} /$ h,p\&lt0.05), maternal grooming in released period was higher than that in control period (control $49.4 \pm 49.9 \mathrm{~s} / \mathrm{calf} / \mathrm{h}$; released $196.5 \pm 23.6 \mathrm{~s} / \mathrm{calf} / \mathrm{h}, \quad p<0.01)$. Pre-weaned calves might have brushgrooming desire and that shared brush-grooming and maternal grooming. 


\section{$\mathrm{P}-24-16$}

The entry pattern of cows to the milking unit in an automatic milking system with free cow traffic

Shigeru Morita, Hiroshi Yamada, Shinji Hoshiba, Michio Komiya, Keiji Takahashi

Food and Environment Sciences, Rakuno Gakuen University

The introduction of automatic milking system (AMS) has been increasing for saving the labor, and for the increment of milk production with frequent milking. The AMS is based on the voluntary entry of cows to an AM unit in free cow traffic system. The objective of the present study was to examine the entry pattern of cow to the milking unit. The data of seven days from a dairy farm included 3,522 entries to an AM unit by 51 cow. The regularity of milking was evaluated by the weekly $\mathrm{CV}$. The average length of the milking interval was 6.6 hour. There were refusals (entries without milking and without reward) in about half of the intervals of milking. The daily number of milking with reward increased with the increase of daily entries to the AM unit. For cows who had a "break and run" pattern of entry to the $\mathrm{AM}$ unit, the number of milking was larger and weekly CV was smaller than that of the cows who had the other entry pattern. It followed that frequent entries and intensive entries in the end period of the interval were needed for high regularity of the intervals.

\section{$\mathrm{P}-24-17$}

Does action potentials generated by pulsed infrared laser irradiation to the cochlea, create sound perception in the brain?

Kotaro Onoe, Yuta Tamai, Suguru Matsui, Shizuko Hiryu, Kohta Kobayasi

Graduate School of Lifeand Medical Sciences, Doshisha University

Optical stimulation is a new neural stimulation method using infrared light. Our recent study showed that the optical stimulation to cochlea nerve of Mongolian gerbil induced compound action potentials. However, the experiment does not actually prove that the optical stimulation to the cochlea created a sensation of sound. In this study, we tried to discover how the action potentials induced by the optical stimulation of cochlea are perceived. We used pulsed-laser train and acoustic-click train with various repetition rate, and observed the Miss Match Negativity (MMN) in the Auditory Evoked Potentials (AEP). We compared AEP evoked by both acoustic and pulsed-laser train stimulation. As results, the MMN amplitude systematically varied with the repetition rate of deviant stimulus, and both stimulation method yielded comparable MMN-amplitude-repetition-rate functions. The data suggests that action potentials induced by optical stimulation in the cochlea are perceived as same as a sound stimuli in the brain. Also stimuli with different repetition rate are perceived as a different pitch sound. Finally, it could be possible to create sound by optical stimulation ignoring place pitch organization.

\section{P-24-18}

Effects of pre-reinforcer and post-reinforcer delays on pigeons' choice: An examination using concurrent-chains schedules

YUMI HATA, DAISUKE SAEKI

Graduate School of Literature and Human Sciences, Osaka City University

The present study examined effects of pre- and post-reinforcer delays on choice in pigeons with a concurrent-chains schedule. Method: Eight male pigeons were used as subjects. Two experimental chambers with two keys on the front wall were used. Subjects were exposed to concurrent-chains schedules as choice situations. In pre-reinforcer delay conditions, three pairs of pre-reinforcer delays ( $2 \mathrm{~s}$ vs. $2 \mathrm{~s}, 2 \mathrm{~s}$ vs. $8 \mathrm{~s}$, and $2 \mathrm{~s}$ vs. $20 \mathrm{~s}$ ) were studied with equal post-reinforcer delays of $2 \mathrm{~s}$. In post-reinforcer delay conditons, three pairs of post-reinforcer delays ( $2 \mathrm{~s}$ vs. $2 \mathrm{~s}, 2 \mathrm{~s}$ vs. $8 \mathrm{~s}$, and $2 \mathrm{~s}$ vs. $20 \mathrm{~s}$ ) were studied with equal pre-reinforcer delays of 2 s. Reinforcer amouts were three pellets for both alternatives. Results and Discussion: An 
analysis of simple main effects of delay conditions indicated that there was a decrease in preference for the larger delayed alternative as the pre-reinforcer delay increased, whereas the preference did not change when the post-reinforcer delay was changed. The present study showed that the pre-reinforcer delay had a greater effect on pigeons' choice than did the postreinforcer delay as in the previous studies on animal choice.

\section{P-24-19}

Long-term change of allopreening between juvenile males and females in captive large-billed crows ERI MiYazaWA ${ }^{1)}$, AyUmi MotaI ${ }^{1)}$, EI-ICH IZAWA ${ }^{2)}$

${ }^{1)}$ Graduate School of Human Relations, Keio University,

${ }^{2)}$ Faculty of Letters, Keio University

Allopreening (an avian analogue of mammalian allogrooming) has been known to occur between pair-bonded male and female in many species of birds, and is thought to play a role in pair-bond maintenance. However, allopreening also occurs both inter- and intrasexually in juvenile groups of large-billed crows (Corvus macrorynchos), in which they spend their first 3-4 years before sexual maturity. We hypothesized that intersexual social interactions, including allopreening, play an important role in pair-bond formation in these juvenile groups, and predicted that the frequency of intersexual allopreening will increase as they are reaching sexual maturity. To test this prediction, we analyzed the direction and the frequency of allopreening in a captive group of juvenile crows, which was formed when they were yearlings. Based on the continuous observational data from 1 to 2.5 years old, we found that males already started to preen females at 1 year old, and its frequency did not change throughout the study period. On the other hand, females started to preen males more at 2 years old. We expect a further increase of intersexual allopreening in the later years of juvenile period, and suggest the importance of female-initiated intersexual interaction in formation of pair bond.

\section{$\mathrm{P}-24-20$}

Investigation of group movement rule in a captive flock of large-billed crows (Corvus macrorhynchos)

AYUMI MOTAI, EI-ICHI IZAWA

Department of Psychology, Keio University

Collective decision making for group movement has been studied across taxa. Two rules are proposed to govern the group movement; despotism and democracy. In mammals, wild baboons despotically follow the choice of specific leaders, while red deer democratically move when approximately 60\% individuals of the herb stand up. In birds, dominant species of mixed-species flocks tended to lead the movement of allospecific individuals. However, there is no study investigating the mechanism governing group movement at the individual level in birds. In this study, we examined which rules, despotism or democracy, could govern the flock movement in a captive flock of sub-adult large-billed crows. The 10 birds $(5$ males and 5 females) were introduced into one of two rooms which were divided an aviary into half. Identity, order, and latency of each bird moving to another room were recorded. We found three types of individual behaviour: one (or two) specific individuals moving first, the immediate follower, and the independent individuals moving with a certain delay to the first individuals. Our finding suggests the two rules might be contaminated in the flock movement in this captive population.

\section{$\mathrm{P}-24-21$}

Effects of neonatal oxytocin administration on socio-emotional behavior in pubertal mice

\section{SAORI Ebara, TOMOKO UeKiTA, TOSHIRo SAKAmoto}

Department of Psychology, Kyoto Tachibana University

Oxytocin plays important roles in regulating social behavior. Here, we examined the effects 
of neonatal oxytocin administration on social conditioned place preference (SCPP) and anxietyrelated behaviors in pubertal mice. Oxytocin, an oxytocin antagonist, or saline was injected (intraperitoneally) into mice within $24 \mathrm{~h}$ of birth. When the mice reached puberty at 29-30 days old, we conducted three types of behavioral tests: SCPP, light-dark, and elevated zero maze. For SCPP, we found that mice given oxytocin showed a preference for the place they had stayed with a cage mate during the conditioning session, whereas mice receiving either the oxytocin antagonist or saline showed no preference. This result suggests that neonatal oxytocin administration enhances place preference for a socially conditioned context in pubertal mice. In the light-dark test, mice given oxytocin or the oxytocin antagonist remained in the light box longer than those given saline did. By contrast, no significant differences were found among the three groups for anxiety-related behavior in the zero maze test. Thus, the effects of oxytocin treatments on the light-dark and zero maze tests were different. Therefore, the relationship between oxytocin and emotionality remains unclear and further study is needed.

\section{P-24-22}

Female preference for male vocalizations in $\mathrm{C} 57 \mathrm{BL} / 6 \mathrm{~J}$ mice

Yui MATSUMOTO ${ }^{1,2)}$, AKARI ASABA ${ }^{1,3)}$, KOUTA KANNO ${ }^{3,4)}$, TAKEFUmi KIKUSUI ${ }^{3)}$, KAZUO OKANOYA ${ }^{2)}$

${ }^{1)}$ National Institute of Neuroscience, National Center of Neurology and Psychiatry,

${ }^{2)}$ Graduate School of Arts \& Science, The University of Tokyo,

${ }^{3)}$ Graduate School of Veterinary Medicine, Azabu University,

${ }^{4}$ Graduate School of Humanistic-Sociological Sciences, Kagoshima University

Male mice produce complex sounds with high frequency in male-female interaction. Recently, I found that the male vocalizations are consisted of several patterns and producing of each pattern is specific to a phase of male physical courtship behavior. This result suggests that the phase-specific pattern of vocalizations has an important role to switch to the next phase. In the present study, we investigate preferences for male vocalizations in female mice using two-choice test to examine the role of each pattern of vocalizations for male-female interaction. As a result, sexually naive females preferred simple pattern of vocalizations that was produced in early-phase of male courtship behavior, to complex ones that was produced in latter-phase of the behavior. On the other hand, the preferences in sexually experienced females varied. These results therefore indicated that preferences for the male vocalizations during courtship were changed according to sexually experiences in females, suggesting that complexity of male vocalizations is associated with mate choice in females.

\section{$\mathrm{P}-24-23$}

The effect of deadlines on the performance of the ladder rung walking task in rats YAYOI SEKIGUCHI ${ }^{1)}$, TOSHIMICHI HATA ${ }^{2)}$

${ }^{1)}$ Graduate School of Psychology, Doshisha University,

${ }^{2)}$ Faculty of Psychology, Doshisha University

We examined the effect of deadlines on the performance in the ladder rung walking task (Metz \& Whishaw, 2002) with two experiments. Rats were trained to cross the horizontal ladder for food rewards. A buzzer tone or a cooing tone was presented for $25 \mathrm{~s}$ beginning when the rats passed the starting point. If they could not reach the goal point before the termination of the tone presentation, rewards were not provided. In the test phase, the duration of one of the tones remained at $25 \mathrm{~s}$ (FREE condition). The other tone duration was shortened to the longest run time of each rat at baseline (around $2.5 \mathrm{~s}$ ) for three sessions (LIMITED condition). In the experiment $1(n=5)$, the run time was increased and slipped steps were decreased in the FREE condition. In the experiment $2(n=10)$, such differences were not observed. Subjects of experiment 1 run more slowly and tend to made more errors at the baseline than the 
subject of Experiment 2. These results showed that the degree of expertise at baseline may interact with the effect of the deadline.

\section{P-24-24}

Individual ability for fear extinction is independent from the depressive-like behaviors in rats MASAHARU UENO ${ }^{1,2)}$, KAZUO YAMADA ${ }^{3)}$, YUKIO ICHITANI ${ }^{3)}$

${ }^{1)}$ Graduate School of Comprehensive Human Sciences, University of Tsukuba,

${ }^{2)}$ JSPS Research Fellow, ${ }^{3)}$ Faculty of Human Sciences, University of Tsukuba

Some individuals recover quickly from stressful events while others do not, suggesting individual differences in stress resilience and vulnerability. Recent studies have shown that individual ability to extinct conditioned fear would predict stress resilience in rats. In the present study, we investigated whether resilient or vulnerable individuals in fear conditioning would show low or high depressive-like behaviors, respectively. Male Wistar-Imamichi rats were subjected to a series of behavioral paradigms, including a sucrose preference test, a forced swim test, and auditory fear conditioning. The subjects were divided into the resilient and vulnerable groups based on their freezing levels at the third extinction trial, and their depressive-like behaviors were compared. Rats in the resilient group showed significant faster extinction of conditioned fear than rats in the vulnerable group. On the other hand, those individual phenotypes were not correlated with the sucrose consumption in the sucrose preference test and the time spent immobile in the forced swim test, both of which are thought to be indices of the level of depression. These results suggest that resilience in a stressful situation is independent from that in depression.

\section{$\mathrm{P}-24-25$}

Rats do not suppress intake of less preferable food in anticipation of more preferable food Mikita NishiKaWA, TOHRU TANIUCHI

Kanazawa University

Flaherty and Checke (1982) found that rats refrained from drinking a less preferable saccharin solution in anticipation of a more preferable sucrose solution given 30 min. later. The purpose of the present study was to reexamine findings of Flaherty and Checke (1982) and to examine effects of some experimental factors. In Experiment 1, the Experimental group was presented with a $0.15 \%$ saccharin solution first and then a $32 \%$ sucrose solution 30 min. later. Control rats were presented only with the saccharin solution. Any reliable difference in amount of intake of saccharin in both groups was not found. Contrary to the prediction, shortening of saccharin-sucrose interval to $5 \mathrm{~min}$. increased intake of a saccharin solution in the Experimental group. In Experiments 2, 3, and 4, although we examined effects of types of experimental apparatus and preliminary training, and levels of food or water deprivation, any suppression of saccharin intake in the Experimental group could not be observed. Discrepancies of results and procedures between the present study and Flaherty and Checke (1982) will be discussed.

\section{$P-24-26$}

Effects of inter-presentation interval in the sample phase and retention interval on the temporal order memory using spontaneous object recognition paradigm in rats

\section{TAICHI HATAKEYAMA ${ }^{1)}$, KAZUO YAMADA ${ }^{2)}$, YUKiO ICHITANI ${ }^{2)}$}

${ }^{1)}$ Graduate School of Comprehensive Human Sciences, University of Tsukuba,

${ }^{2}$ Institute of Psychology and Behavioral Neuroscience, University of Tsukuba

To investigate how long rats can retain memory of temporal order of their experience, temporal order memory using spontaneous object recognition (SOR) was analyzed. The test consisted of a sample phase, a retention interval $(3-30 \mathrm{~min})$ and a test phase $(5 \mathrm{~min})$. The sample phase consisted of 5 presentations with different objects. In a presentation, two 
identical objects were placed in an open field and rats explored them for $5 \mathrm{~min}$. The interpresentation interval was $3 \mathrm{~min}$. After the retention interval of 3,10 , or $30 \mathrm{~min}$, the test phase started. In the test phase, the second and fourth objects were placed. After the retention interval of $3 \mathrm{~min}$, rats explored the old (second) object more than the recent (fourth) object, but after 10 and $30 \mathrm{~min}$ interval, they explored equally two objects. However, with longer inter-presentation interval (10 and $30 \mathrm{~min}$ ), rats could explore the old (second) object more than the recent (fourth) object even after longer retention interval of $10 \mathrm{~min}$. Results suggest that rats can retain temporal order memory for 10 min depending on the length of interpresentation interval.

\section{$\mathrm{P}-24-27$}

\section{Effects of social interactions on spatial learning in a lattice maze}

NOBUYa SATO, SAKI ISHII

Department of Psychological Sciences, Kwansei Gakuin University

To examine the effects of social interactions on spatial learning, we trained rats to perform a route learning task in a lattice-shaped maze. The rats latently learned the spatial layout of the lattice maze by exploring the maze for three days before the learning session. We divided rats into three group: dyad, single, and control groups. The rats in the dyad group explored the maze in pairs. The rats in the single group explored the maze by itself. The control group never explored the maze. In the route learning session, the rats were required to go from a start box to a goal box along with a pre-determined route in the maze. The time and the number of passing segments in the maze to reach the goal box were measured. As a result, the time to reach the goal decreased gradually in the route learning. The number of passing segments approached to the smallest number required to reach the goal. The group differences were found in the velocity of rats' run. This may suggest the effects of the social interaction on spatial learning. The length of the exploring period was discussed.

\section{$\mathrm{P}-24-28$}

Tool manipulation by rats according to food position

AKANE NAGANO ${ }^{1)}$, KenJiRo AOYAMA ${ }^{2)}$

${ }^{1)}$ Graduate School of Psychology, Doshisha University,

${ }^{2}$ Faculty of Psychology, Doshisha University

We investigated whether rats could move a tool laterally in relation to the position of food without the experience of having performed this action during training. The rats were trained to use rake-shaped tools to obtain food beyond their reach. In the training, they had to choose between an appropriately arranged rake with food placed inside it, and an inappropriately arranged rake with food placed either outside the rake or nowhere near the rake. If the rat pulled the appropriate rake directly towards itself, it could obtain the reward because the food was placed inside the rake. On the other hand, the rat pulled the inappropriate rake, it could not obtain the reward. The rats never moved the rakes laterally to the food during the training. In the test, the rake was placed at the center of the apparatus, and the food was placed on either side of the rake. Hence, the rats had to move the tool laterally before pulling it. Three out of four rats were able to move the rake laterally according to the position of the food.

P-24-29

Cats' vocalization toward owners across different situations and contexts MinORI ARAHORI ${ }^{1,2)}$, YUTA SHINYA ${ }^{2,3)}$, KAZUO FUJITA ${ }^{1)}$

${ }^{1)}$ Graduate School of Letters, Kyoto University, ${ }^{2)}$ JSPS Research Fellow,

${ }^{3)}$ Graduate School of Education, Kyoto University

Companion animals appear to have acquired excellent ability to communicate with humans 
through the process of domestication, and studies revealed differences in communicational strategies between domesticated animals and their ancestors. For example, domestic cats (Felis catus) vocalize more pleasantly than wild cats (Felis silvestris lybica) from human perspective (Nicastro, 2005). However, few studies have examined how flexibly companion animals use their vocalization depending on different situations and contexts. In this study, we asked owners keeping one cat to record their cats' greeting vocalization when the owners came back home, and their purring vocalization whenever they apparently claimed food or play from owners. We also collected the following information of how much food remained in the bowl and whether other family members were already at home, and when owners left and came back home. The owners also answered the cat version of the Monash Dog Owner Relationship Scale (Dwyer et al., 2006) to measure owners' attachment to their pets. Acoustical analysis of duration of calls, fundamental frequency, and the first and second formant was conducted to reveal the vocal differences among situations, and the effect of contexts. The results will be reported at the meeting.

\section{$\mathrm{P}-24-30$}

The positive reinforcement of owner's gaze to dogs

Midori OhKita ${ }^{1)}$, Miho Nagasawa ${ }^{2,3)}$, Kazutaka Mogi ${ }^{2)}$, Takefumi Kikusui ${ }^{2)}$

${ }^{1)}$ School of Human Sciences, Senshu University,

${ }^{2)}$ School of Veterinary Medicine, Azabu University,

${ }^{3}$ School of Medicine, Jichi Medical University

Although the recent research showed that human gazes increased dog's social behaviors (Nagasawa et al., 2009, 2015; Ohkita et al., 2016), there was no direct evidence that human gaze act as a positive reinforcement for dogs. In the present study, to assess positive reinforcement property of owner's gaze, simple operant conditioning situation was conducted. There were two conditions: Direct Gaze and Avert Gaze condition. In Direct Gaze condition, a spontaneous response, which dog's nose went across an imaginary line, was followed by a direct gaze photo of the owner on a display. In contrast, in Avert gaze condition, it was followed by an avert gaze photo of her/him. Each condition was consisted of three blocks ( $5 \mathrm{~min} / \mathrm{block})$. All pairs of the dogs and the owners conducted both conditions. The results showed that the responses decreased through the blocks in Avert Gaze condition. In contrast, they slightly increased through the blocks in Direct Gaze condition. There was significantly difference in 3rd block between the conditions. Therefore, owners' direct gazes might increase the spontaneous behavior of the dogs. We suggest that human gazes act as a positive reinforcement for dogs, and may contribute to close relationships between humans and dogs.

\section{$\mathrm{P}-24-31$}

Can we estimate dogs' recognition of objects in mirrors from their behaviour and response time? MEgumi FuKuZaWA, AyANO HASHI

College of Bioresource Sciences, Nihon University

Our aim was to examine dogs' behaviours to investigate how they recognise objects visible in mirrors. Ten pet dogs that had never undergone mirror exposure testing were recruited and categorised into two groups; a habituation-to-the-mirror group (HG) and no-habituation group (NHG). The HG was given time to explore the test room before each test. A food reward was placed in a bowl positioned on the far side of an opaque barrier. The dog was able to confirm the presence of the reward via the mirror but was unable to confirm it directly. Both the time taken for the dog to get the reward and the dog's behaviours in obtaining it were recorded. All dogs achieved the criterion, and the time they took to get the reward was shortened significantly with repetition, irrespective of group. However, both the time taken to reach the reward and the time spent looking in the mirror were significantly shorter in the HG than in the NHG. These results suggested that experience in habituation with a mirror 
effected an exploratory response in the dogs, and also indicated that dogs were able to generalise the mirror silhouette and to use them without human communicative cues.

\begin{abstract}
P-24-32
Influence of social interactions with unrelated adult females on development of vocal greeting behaviors in Japanese macaques

NORIKO KATSU, KAZUNORI YAMADA, MASAYUKI NAKAMICHI

Department of Human Sciences, Osaka University

We aimed to clarify how social interactions with unrelated adult females affect development of greeting behaviors of Japanese macaques (Macaca fuscata), who live in large social group. We focused on greeting vocalizations, 'grunts' and 'girneys'. These vocalizations function to smooth social interactions by communicating non-hostle intent of callers. We conducted focal observation on 30 females from infancy to full adulthood in Arashiyama group, a free-ranging group of Japanese macaques. We found that adult females emitted the vocalizations more frequently when approaching to unrelated adult females than related ones, whereas this difference was not shown in young adult and juvenile females. Females older than juveniles were more likely to engage in affiliative interactions such as grooming after approaching unrelated females with vocalizations than without vocalizations, indicating that vocal use can be beneficial for callers. Young adult females who have experienced more social interactions with unrelated adult females emitted the vocalizations more frequently when approaching to unrelated adult females in the following year. Experiences of social interactions with related adult females did not influence vocal greeting behaviors of young adult females. These findings suggest that social experience with unrelated adult females modifies vocal greeting behaviors which promote social interactions.
\end{abstract}

\title{
$\mathrm{P}-24-33$
}

Serial learning of Arabic numerals and working memory in a captive infant western gorilla MASAYUKI TANAKA ${ }^{1,2)}$, MitsunOri NAGAO ${ }^{1)}$, AKIHIRo MizUnO ${ }^{1)}$

${ }^{1)}$ Center for Research and Education of Wildlife, Kyoto City Zoo,

${ }^{2)}$ Wildlife Research Center, Kyoto University

Cognitive tasks were given to an infant western gorilla (Gorilla gorilla) in Kyoto City Zoo. These were intended for not only the comparative cognitive studies but also cognitive enrichment of participants. These tasks were serial learning and working memory tasks using Arabic numerals. The subject learned rapidly and acquired the correct number sequence from 1 to 13 in a period of one and half year. Besides learning the correct sequence, the gorilla infant also learnt a "working memory task" (cf. Inoue and Matsuzawa, 2007). As part of the task, when the subject touched "1," the remaining numerals were masked with checkered squares. The gorilla infant was able to choose the correct sequence from 1 to 5 . He chose the correct sequence in $80 \%$ of the trials. He stepped up to the new sequence from 1 to 6 . In every step of the task, he started from low-level performance and then acquired a higher level of performance through learning. These results suggest that the gorilla infant did not see the whole sequence from the beginning, but learned to see it as the task progressed.

$\mathrm{P}-24-34$

Public attitude for evacuation with pets under emergency disaster circumstances

Chika Matsumoto ${ }^{1)}$, Kiriko SAKATA ${ }^{1)}$, Hitomi SugiURA ${ }^{2)}$

${ }^{1)}$ Graduate School of Integrated Arts and Sciences, Hiroshima University,

${ }^{2)}$ College of Sport and Health Science, Ritsumeikan University

This study was designed to identify psychological variables of the general public that could facilitate or inhibit the evacuation with pets and living with pets in shelters or temporary housing during emergencies. An Internet-based survey was conducted in 2015 focusing on 
differences in attitudes between pet owners and non-owners $(\mathrm{N}=600)$. Multiple regression analysis was conducted with "Co-evacuation with pets" and "Willing to accept others' pets during evacuation and in shelters" as objective variables. Results indicated that pet owners' "Worrying about around the other people" did not significantly inhibit co-evacuation. Moreover, approval of pets among pet owners aware of Ministry of the Environment recommendations was significantly higher than among unaware pet owners. Furthermore, "Worrying about around the other people" by people without pets had a significantly negative effect on each objective variable. Also, "Treating pets as equal to humans", or having an "Equal status" had a significantly positive effect on the objective variables. These results suggest that the treatment of pets as equals by pet owners might be one factor facilitating positive attitudes in people without pets about co-evacuation with pets and having others' pets during evacuations and in shelters.

\section{$\mathrm{P}-24-35$}

The Japanese monkey prefers gentle stroking to hand clapping in an overcoming-difficulties procedure

MARI KUMASHIRO, KAZUYUKI SAMEJIMA

Brain Science Institute, Tamagawa University

It is unknown which modality is effective in a multimodal social-interaction between monkeys and humans. We aimed to test which of two treatments, touch or sound, was useful in an overcoming-difficulties procedure with a single subject design by a female Japanese monkey weighing about $6 \mathrm{~kg}$. The experimenter encouraged her to eat the least favorite food, carrots, by stroking the face (touch-treatment) or by clapping (sound-treatment). In the soundtreatment, the throwing-food problem behavior remarkably increased. The treatment by hand clapping seems to interfere with a stable relationship. Our data show that the monkey preferred the touch-treatment to the sound-treatment in the overcoming-difficulties procedure. Touch may be a useful modality to have a positive social-interaction with monkeys.

\section{$\mathrm{P}-24-36$}

Do primate models of autism care other individuals when they receive less valuable reward than other individuals?

\section{MiYUKI YASUE ${ }^{1)}$, AKIKO NAKAGAMI ${ }^{1,2)}$, KeIKo NAKAGAKI ${ }^{1)}$, NORITAKA ICHINOHE ${ }^{1)}$,} NOBUYUKI KAWAI ${ }^{1,2)}$

${ }^{11}$ Department of Ultrastructural Research, National Center of Neurology and Psychiatry,

${ }^{2)}$ Graduate School of Information Science, Nagoya University

We previously showed that marmosets discriminated between human actors who reciprocated in social exchanges and those who did not (Kawai et al., 2014). In the reciprocal condition, two actors exchanged food equally, while in the non-reciprocal condition, one actor (non-reciprocator) ended up with all food and the other actor with none. After observing these exchanges, the control marmosets avoided receiving food from the non-reciprocator in the nonreciprocal condition. Nevertheless, marmosets with fetal exposure to valproic acid (VPA marmosets) did not (Yasue et al., 2015). The VPA marmosets had showed impaired social interactions in conventional test batteries, inflexibilities in a successive reversal learning test, and limited repertoires of vocal communications. In this study, we investigate whether the VPA marmosets don't notice unbalanced interaction per se or they didn't discriminate thirdparty's balanced and unbalanced interactions because those interactions were irrelevant to the VPA marmosets. We trained marmosets to hold a spoon for more than $2 \mathrm{sec}$. Then they were received either value-matched or less valuable reward than the reward received by the visible conspecifics. We accounted frequency that VPA marmosets rejected the lower valuable reward or avoided to hold the spoon, negative reaction for unequal rewards. 
$\mathrm{P}-25-01$

Adaptive adjustment of frequency structure of echolocation sounds in bats during group flight with conspecifics

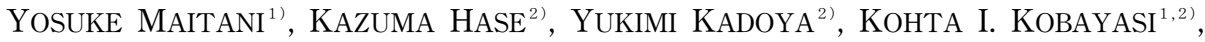 \\ SHIZUKO HIRYU ${ }^{1,2,3)}$ \\ ${ }^{1)}$ Faculty of Life and Medical Sciences, Doshisha University, \\ ${ }^{2)}$ Graduate School of Life and Medical Sciences, Doshisha University, ${ }^{3)}$ JST, PRESTO
}

Bats emit ultrasound and listen to the returning echoes to localize insect prey and avoid obstacles. When they fly with multiple conspecifics, the sounds emitted by the other bats and their own echoes create acoustic interference. Here, we examined how bats (Miniopterus fuliginosus) changed their acoustic characteristics of emitted pulses when flying in groups. Miniature telemetry microphone was mounted on individual bats' back so that echolocation pulses could be recorded separately during group flight. As a result, we found that the bats shifted their terminal frequency (TF) of downward frequency modulated (FM) sounds away each other during group flight, whereas they did not do so during single flight, indicating that they actively weaken the similarity of the echolocation sounds among individuals flying together. Furthermore, we conducted additional experiment in which artificial downward FM jamming sounds (linear FM sounds and exponential FM sounds) were presented by four loudspeakers to the bats flying alone. No significant change was observed for the linear FM sounds but the bats raised the TFs by $1-3 \mathrm{kHz}$ responding to the exponential $\mathrm{FM}$ sounds. These findings suggest that bats adaptively adjust the TF to avoid the sounds similar to their own during echolocation.

\title{
$\mathrm{P}-25-02$
}

Comparison of time perception using the interval bisection task with continuous tone and pulse tone in rats

Yuta NAKAMURA ${ }^{1)}$, Shogo SAKATA ${ }^{2)}$

${ }^{1)}$ School of Integrated Arts and Sciences, Hiroshima University,

${ }^{2)}$ Graduate School of Integrated Arts and Sciences, Hiroshima University

We often experience that the objective same time is felt subjectively long or short. This study investigated the effects of the stimulus property using the temporal bisection task in rats. During the first 20 days the rats learned to press the left lever following a 2-sec tone and the right lever following an 8-sec tone. The first presentation stimulus was continuous tone at training 20 sessions and the following 5 days bisection test sessions. And then $4 \mathrm{~Hz}$ pulse tone was presented at 5 days bisection test. These data plot as a logistic curve. The signal duration that was associated with 50\% long responses was calculated and reported as the point of subjective equality (PSE). The first training stimulus was continuous tone and the PSE of the continuous tone test was shorter than that of the $4 \mathrm{~Hz}$ pulse tone test. Then the second training stimulus was $4 \mathrm{~Hz}$ pulse tone. And following test sessions were likely as describe before only change the stimulus presentation order. The PSE of the $4 \mathrm{~Hz}$ pulse tone test was shorter than that of the continuous tone one. These results suggest that the training stimulus property have an influence for the time perception directly.

$\mathrm{P}-25-03$

Audio-visual integration in rodent

Yuki ITO $^{1)}$, Ryo SATO ${ }^{1)}$, TAKahumi Huruyama ${ }^{1,2)}$, Shizuko Hiryu ${ }^{1,3)}$, Kota Kobayashi ${ }^{1)}$

${ }^{1)}$ Life and Medical Science, Doshisha University,

${ }^{2)}$ Reserch fellow of Japan society for the promotion of Science, ${ }^{3)}$ JST PREST

Multimodal integration in human is well known, but less is known in other species. This study investigated whether audio-visual integration occurs in rodent (Mongolian gerbil) by using both behavior and neurophysiological technique. Experiment I investigated whether the 
double-flash illusion occurs by novel object recognition procedure. An object was set in a free field; a light-emitting diode was embedded in it. In habituation trials, gerbils explored a flashemitting object, while the double tone pips were presented from the speaker. The onset of flash and sound were not synchronized until test trial. As a result, gerbils explored the object more in test trial. These results suggest that double-flash illusion occurs in gerbil. Experiment II investigated audio-visual integration by cortical evoked potential. Visual stimulus was presented either paired with auditory stimulus or unimodally. We recorded the evoked potential from primary visual cortex (V1) and surrounding regions of gerbils. As a result, multimodal enhancement of VEP's peak amplitude was observed only around V1. These results suggest that visual stimulus is influenced by auditory stimulus in the area. Our data indicates that audio-visual integration occurs in gerbils and the neural mechanism of integration can be unraveled by using the animal.

\section{$\mathrm{P}-25-04$}

\section{Lateralized courtship display in Java sparrows}

RIKA ENDO ${ }^{1)}$, MASAYO SOMA ${ }^{2)}$

${ }^{1)}$ Graduate School of Life Science, Hokkaido University,

${ }^{2)}$ Faculty of Science, Hokkaido University

Behavioral and visual lateralization is seen in many animals, and generally is assumed to be adaptive as it enhances performance. According to past studies, vertebrates show biased eye use for seeing conspecifics or predators. Body motions associated with communicative behavior are also lateralized in some species. In birds, a few studies focused on visual laterality in the context of courtship and mate choice, and found that biased eye use is crucial for males to choose right partners. However, such visual laterality is not preserved among species. Moreover, previous studies lack insight into whether females show similar visual laterality with males. In this aspect Estrildid fiches are interesting subjects because they show courtship display when a male and a female sit side by side, which inevitably poses behavioral/visual laterality. We studied a species of Estrildid finch, Java sparrow, Lonchura oryzivora. We observed Java sparrow pairs using video cameras, and examined whether their courtship behavior and eye use showed any side-bias, specific to sex or individuals. As a result, we found large among- and within- individual variations in laterality, but non-significant sex differences. We will discuss these findings in relation to courtship process and copulation success.

\section{$\mathrm{P}-25-05$}

$A$ rubber tail task in CAPS2 KO mice: second report

MAKOTO WADA $^{1)}$, MASAKAZU IDE ${ }^{1,2)}$, TAKESHI ATSUMI ${ }^{1)}$, KAORI YAGISHITA ${ }^{3)}$, MOMOKO KATAKAI $^{3)}$, Yo SHINODA ${ }^{3,4)}$, TEIICHI FURUICHI ${ }^{3)}$, KENJI KANSAKU ${ }^{5,6)}$

${ }^{11}$ Department of Rehabilitation for Brain Functions, Research Institute of NRCD,

${ }^{2}$ JSPS Research Fellow,

${ }^{3)}$ Faculty of Science and Technology, Tokyo University of Science,

${ }^{4)}$ Department of Environmental Health, School of Pharmacy, Tokyo University of Pharmacy and Life Sciences,

${ }^{5}$ Department of Rehabilitation for Brain Functions, Research Institute of NRCD,

${ }^{6}$ Brain Science Inspired Life Support Research Center, The University of ElectroCommunications

Dysfunctions of body image are often observed in persons with autism spectrum disorder (ASD); however, such cognitive functions have not been evaluated in mouse model of ASD. We have developed a rubber tail task in mice by stroking a real tail and a rubber tail, aiming to evaluate dysfunctions of body image, and found that the rubber tail response was not obvious in CAPS2 KO mice, which show autistic-like phenotypes; but response rates were generally 
low in the CAPS2 KO mice, compared with wild type mice. In present study, we investigated responses of tail grasping in the CAPS2 KO mice and wild type mice. Without any additional tactile stimuli, real tails or rubber tails were grasped by an experimenter. When the rubber tails were grasped, response rates were significantly low in the CAPS2 KO mice. In contrast, similar responses to the wild type mice (e.g., orienting or retracting the head) were observed in the KO mice, when the real tails were grasped; response rates of the $\mathrm{KO}$ mice were comparable. The present result enhances our interpretation that dysfunctions in body ownership in ASD were partly mimicked in the mouse model of ASD.

\section{$\mathrm{P}-25-06$}

\section{Acoustic event-related potentials in Rat's communications} YUMI SAITO, KAZUO OKANOYA

The Graduate School of Arts and Sciences, The University of Tokyo

Rats (Rattus norvegicus) emit $50-\mathrm{kHz}$ ultrasonic vocalizations (USVs) in order to serve a communicative purposes to conspecifics, such as play, mating and approach for the novel individual. They also show $22-\mathrm{kHz}$ communicative USVs as the alarm calls, so we hypothesized their brain activities show the selectivity to these specific bandwidth. Eventrelated potentials (ERPs) in rat's prefrontal cortex were recorded when hearing some pure tones which have various frequencies. In results, mean amplitudes of some ERP components (P1, N1, P2 and N2) coincided with the loudness curve of arbino rats. Especially, large amplitudes were shown in presentation of $40-50 \mathrm{kHz}$ pure tones. Results suggested that these ERP components reflected acoustic sensitivities of rats, including $50-\mathrm{kHz}$ communicative vocalizations.

\section{P-25-07}

The effect of timing of maternal separation on isolation-induced ultrasonic vocalization in rat pups

YURIE KAWAMURA, HiROMI WADA

Graduate School of Letters, Hokkaido University

Maternal separation affects the interaction between a rat dam and its pups. Rat pups emit ultrasonic vocalizations (USVs) when they are separated from their dam and these USVs induce maternal behavior in their dam. Since maternal separation chronically deprives pups of maternal care, it is considered a stressor that affects USVs. However, little is known about the effect of maternal separation on USVs. We compared the effects of early (postnatal days 4-9), middle (postnatal days 10-15) and late (postnatal days 16-21) maternal separation on pup USVs to a non-maternal separation control group. Since previous studies reported that frequency-modulated USVs are more important for mother-infant communication than other USVs, we classified frequency modulated USVs into four types: up, down, flat, and others based on the waveform of the USVs. Rat pups exposed to early maternal separation showed increased number of up type USVs compared with other pups. This indicates that early maternal separation affects mother-infant communication due to the increases of up type USVs. Furthermore, our results suggest the existence of a critical period of mother-infant interaction for the development of USVs. 
${ }^{1}$ Bio-Laboratory, Foundation for Advancement of International Science,

${ }^{2)}$ Graduate School of Health and Welfare Sciences, International University of Health and Welfare,

${ }^{3)}$ Department of Food and Nutrition, Tokyo Kasei University,

${ }^{4)}$ Department of Pharmaceutical Sciences, International University of Health and Welfare

Adolescence, the transition period between childhood and adult life, is a critical period for neural and social development. The maturation of dopaminergic neurotransmitter systems, particularly in the prefrontal cortex and limbic regions, also occurs during adolescence, and determines the sensitivity to rewarding effects of food and drugs, etc. Additionally, social interactions are important for neuronal and behavioral development. Meanwhile, adolescent rats emit $50-\mathrm{kHz}$ ultrasonic vocalizations (USVs), a marker of positive emotion, during social play with its mates, which is referred to as rough-and-tumble play. We recently showed that tickling, which mimic rough-and-tumble play with human hand, triggers dopamine release with $50 \mathrm{k}-\mathrm{Hz}$ USVs in the nucleus accumbens, and tickling-induced $50-\mathrm{kHz}$ USVs were inhibited by the direct microinjection of dopamine receptor antagonist into the nucleus accumbens. In the present study, we investigate whether there are differences between D1 and D2 receptor subtypes antagonism to response by tickling in the nucleus accumbens. As a result, D1 or D2 receptor antagonism alone altered the production of $50 \mathrm{kHz}$ USVs, but also these dopamine receptor subtypes appear to influence acoustic parameters to different degrees. These results may indicate that during adolescence the potentially different contributions receptor subtypes play in positive emotion control in the nucleus accumbens.

\section{P-25-09}

Do stroking stimuli induce a pleasant sensation in rats

Shota OKabe, Yuki Takayanagi, Masahide Yoshida, Ayumu Inutsuka, TATSUSHI ONAKA

Department of Physiology, Jichi Medical University

Gentle stroking induces a pleasant sensation when servers and receivers of stroking stimuli have an affiliative relationship. Neural mechanisms underlying relationship-dependent responses to stroking stimuli unknown. Here, we report procedures of repetitive application of stroking stimuli to form an affiliative relationship. Female rats $(\mathrm{LEW} / \mathrm{CrlCrlj}$ ) received 5-min stroking stimuli by use of experimenter's hand every other day for 4 weeks between 3 and 7 weeks of age (S3-7 group), for 4 weeks between 7 and 11 weeks of age (S7-11 group), or for 8 weeks between 3 and 11 weeks of age (S3-11 group). Control rats received no stroking stimuli (N3-11 group). Rats that had received stroking stimuli (S3-7 and S3-11 groups) emitted higher number of $50-\mathrm{kHz}$ ultrasonic vocalizations, an index of positive emotion, more frequently during stroking stimuli. In addition, rats of S3-7, S7-11, and S3-11 groups showed a preference to stroking stimuli in a conditioned place preference test and showed a higher preference for hand of experimenter over a bottle as compared to N3-11 group in object preference test. These findings suggest that procedures of post-weaning stroking procedures induce pleasantness in rats and are consistent a view that post-weaning stroking develops an affiliative relationship between rats and an experimenter. 
$\mathrm{P}-25-10$

Dorsal hippocampal NMDA and AMPA receptor blockade impairs rats' temporal order memory in radial maze

Manami Sugita, KaZuo Yamada, Yukio IChitani

Faculty of Human Sciences, University of Tsukuba

We investigated the involvement of dorsal hippocampal N-methyl-D-aspartate (NMDA) and $\alpha$-amino-3-hydroxy-5-methyl-4-isoxazolepropionic acid (AMPA) receptors in rats' temporal order memory using a radial arm maze. One trial of the task consisted of a study and a test phases. In the study phase, five arms were opened sequentially and the rat received a food reward at the end of each arm. The used arms in each trial and the order of arm presentation were at random. In the test phase that started 1 min after the study phase, two arms that were presented second and forth in the study phase were opened simultaneously. When the rat chose the arm presented earlier in the study phase, it could eat a food reward. After animals reached the criterion, the drug test was conducted. An NMDA receptor antagonist AP5 (20 or $40 \mathrm{mM}$ ), an AMPA receptor antagonist NBQX (5 mM) or vehicle (PB, $0.02 \mathrm{M})$ was bilaterally injected into the dorsal hippocampus $(1.0 \mu \mathrm{l} / \mathrm{side}) 10 \mathrm{~min}$ before the study phase. Both AP5 and NBQX injections significantly disrupted performance of the task. Result suggest that the dorsal hippocampal NMDA and AMPA receptors play an important role in rats' temporal order memory.

\section{$\mathrm{P}-25-11$}

\section{Oxytocin enhances rat's helping behavior for stranger}

ATSUHito YAMAGISHI ${ }^{1)}$, NoBUYA SATO ${ }^{2)}$

${ }^{1)}$ Graduate School of Humanities, Kwansei Gakuin University,

${ }^{2)}$ School of Humanities, Kwansei Gakuin University

We examined the influence of the neuropeptide oxytocin on rat's helping behavior. The rats learned for 20 days to open a door to help a cagemate soaked with water. The rats were divided into familiar and stranger groups. The familiar group had been housed with the cagemate for 14 days before the helping task, and the stranger group had been housed alone. Both the groups had received oxytocin $(1 \mathrm{mg} / \mathrm{kg})$ or saline intraperitoneally injection for consecutive 5 days before the helping task. As a result, more rats in the stranger group with the oxytocin injection showed the helping behavior than the other 3 groups. This result suggests that the oxytocin enhances rat's helping behavior to a stranger, but not to a familiar individual.

\section{$\mathrm{P}-25-12$}

Social influence of a conspecific peer on domestic dogs

ZHANG CHEN, YAMAMOTO SHINYA

Graduate School of Intercultural Studies, Kobe University

Previous studies have shown that domestic dogs (Canis familiaris) use social information provided by human. However, it remains to be investigated whether and how dogs are affected by conspecifics. The aim of this study is to further investigate social influence of conspecifics on their selective behavior during a simple object-choice task. In our experiment, twenty pet dogs were tested in a dog nursery. In the pre-test phase, we confirmed that the participated dog could make a correct choice when it was obvious that which one of two cups contained food. In the test phase, we first evaluated preference of the dogs by allowing them to freely select one of two different-color cups which both contained food. Then we developed two conditions:demonstration and non-demonstration conditions. In the demonstration condition, the dog participants watched a conspecific demonstrating preference for a specific cup, while no choice demonstration was performed in the non-demonstration condition. Finally they were again allowed to select one of two cups freely. In the result, We observed individual 
differences and further investigated factors which may explain the outcome: social relationship between the participants and demonstrator, sociality of the participants and sex.

\section{P-25-13}

Increasing behavioral QOL of the dog

\section{HITOSHI TAKAYAMA}

Graduate School of Science for Human Services, Ritsumeikan University

There is "five freedom" in a guideline of animal welfare for industrial animals, but this is applicable for the companion animal. And behavioral QOL as more better individual measure of QOL (Mochizuki, 2001). Behavioral QOL is measure quality of life with the number of choices of the behavior maintained by positive reinforcement. When the animal has behavior problems, we would to modify the problem by behavior modification or training. However practices emphasizing problem solving can be considered to postpone the positive reinforcement which the animal has right to get. The present study aimed to improve QOL of the "Shiba" dog which dislikes the walking, not by the way of getting rid of aversion itself, but the way of finding the dog's behaviors which the dog is able to do now and which is positively reinforced and by the way of enlarging that behaviors.

\section{$\mathrm{P}-25-14$}

\section{A new non-invasive method to measure cortisol levels from marmoset saliva}

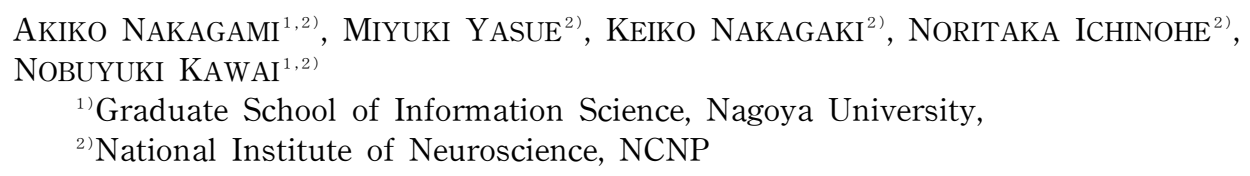

${ }^{2)}$ National Institute of Neuroscience, NCNP

Marmosets are assumed to be susceptible to stressors because of their proportionally larger adrenal gland among primates. Cortisol is an essential hormonal reaction to various stressors, which is elicited by the restoration of basal activity via hypothalamic-pituitary-adrenal (HPA) axis feedback inhibition mechanism. Chronic elevation of cortisol level often leads a pathogenesis of psychiatric disorders such as depression. Although cortisol can be measured in serum, saliva and urine, to evaluate cortisol level accurately measurement per se should not cause stress. We set up a new method to measure cortisol levels from saliva of marmoset without using various flavor and food. This method is simple, but we can collect enough saliva to be analyzed for cortisol. Moreover, to ensure cortisol levels from saliva being accurate, we verified cortisol levels extracted not only from saliva, but also from serum and urine at the same time. We will show this new method and the cortisol levels of marmosets.

\section{$\mathrm{P}-25-15$}

\section{Roles of post-conflict affiliations in bottlenose dolphins} CHISATO YAMAMOTO ${ }^{1,2)}$, TADAMICHI MORISAKA ${ }^{1)}$, KEISUKE FURUTA ${ }^{3)}$, TOSHIAKI ISHIBASHI ${ }^{4)}$, AKIHIKO YOSHIDA ${ }^{5)}$, MichIHIRO TAKI ${ }^{6}$, YOSHIHISA MORI ${ }^{7)}$, MASAO AMANO ${ }^{2)}$

${ }^{11}$ School of Marine Science and Technology, Tokai University,

${ }^{2)}$ Graduate School of Fisheries and Environmental Sciences, Nagasaki University,

${ }^{3)}$ Kobe City Suma Aqualife Park, ${ }^{4}$ Shimonoseki Marine Science Museum,

${ }^{5}$ Kagoshima City Aquarium, ${ }^{6}$ Miyajima Public Aquarium,

${ }^{7}$ Department of Animal Sciences, Teikyo University of Science

Post-conflict affiliations (PCA) may have functions of conflict management, which reduces costs associated with aggressions. However, the functions of these affiliations were little known in non-primates animals. We investigated the effect of PCAs on renewed aggressions, and the relationship between the occurrences of PCAs and an index of affiliative relationships, which calculated from the frequency of affiliation during nonaggressive periods, to understand the roles of PCAs in bottlenose dolphins. Although winners and losers initiated renewed 
aggressions after original aggressions, renewed aggressions decreased after PCA between former opponents, initiated by losers to bystanders, by bystanders toward winners, and by bystanders to losers. There was positive correlation between the occurrences of PCA between former opponents and the index of affiliative relationships between former opponents. There was also positive correlation between PCA by bystanders occurrences and the index of affiliative relationships between opponents and bystanders. These results suggested that PCA between former opponents functioned as reconciliation, and pairs who shared more affiliative relationships tended to reconcile. PCAs by bystanders were suggested to function as appeasement and protection of opponents. Bystanders who have more affilaitive relationships with the opponent may restrain aggressive tendency of opponents by affiliations.

\section{P-25-16}

\section{Dancing chicken and Breland \& Breland: Revisiting the history of animal psychology} MiKI TAKASUNA

School of Social and Human Sciences, Tokyo International University

Just 50 years have passed since publication of Animal behavior (1966) by Keller Breland (1915-1965) and Marian Breland (1920-2001). Five years previously, the Brelands contributed "The misbehavior of organisms," apparently a parody of Skinner's The behavior of organisms (1938). Breland \& Breland (1961) had an enormous impact on the study of learning, such that they left a legacy in the field about aspects of species-specific reactions and constraints on learning. The Brelands were also known for Animal Behavior Enterprises, a company they founded to train animals; animal conditioned behavior was exhibited at various zoos and museums of natural history; was used for department store displays, fair and trade convention exhibits; was presented as entertainment at tourist attractions and on television shows; and used in advertising in the production of television commercials (Breland \& Breland, 1961). To exemplify their popularity, I will show some scenes of a chicken, rabbit, and duck filmed in North Carolina, which appeared in a German film Stroszek (1976) directed by Werner Herzog. The dancing chicken was impressive because it was the first example of the "breakdowns of conditioned operant behavior" in Breland \& Breland (1961).

\section{$\mathrm{P}-25-17$ \\ Interocular independence of attention in pigeons \\ Aya KoKubu, YuYa Hataji, KaZuO FujiTa \\ Graduate School of Letters, Kyoto University}

Pigeons have laterally positioned eyes and 2 foveas in each eye point to different directions. This suggests that they have designed to see at least four positions in their visual field, left front, right front, left lateral and right lateral. An interesting question is whether they actually perceive different visual scenes simultaneously. Kaneko (2013) examined this question by training pigeons on a Posner task (Posner, 1980). In the Posner task, a cue is presented before a target, and subjects are required to react to the target as fast as they can. In Kaneko (2013), a cue and a target was presented either ipsilaterally or contralaterally within or across visual hemifield. The result showed that cueing effect was restricted within the same hemifield. This suggests that pigeons have independent attentional resource for each hemifield. Following this research, we examined whether pigeons have independent attentional resources in the left eye and the right eye, in frontal binocular field. We separated visual input onto both eyes by using anaglyph glasses and conducted a similar Posner task. If pigeons have independent attentional resource for each eye, cueing effect to RT may be restricted within one eye. The result will be presented at the meeting. 
$\mathrm{P}-25-18$

An experimental analysis of the foraging behavior of pigeons in depleting patches using reinforcement schedule controlling energy expenditure

MASANORI KONO ${ }^{1,2)}$

${ }^{11}$ Department of Psychology, Meisei University,

${ }^{2}$ Department of Psychology, Teikyo University

Most optimal foraging theories have been formulated based on the energy expended on the foraging behavior. Therefore, it is necessary to measure the amount of energy related to the foraging behavior of animals in order to investigate the validity of these theories. Previous studies have measured time or number of responses as an alternative of energy. Kono (2013) developed a schedule in which the controlling variable used was energy and showed the utility of the energy schedule as a foraging simulation procedure. The present study employed energy schedules to simulate the foraging situation addressed by the marginal value theorem (Charnov, 1976) and investigated the foraging behavior of pigeons. The results showed that the energy expenditure spent in a patch increased with the energy expenditure required for traveling to other patches as predicted by the marginal value theorem. Additionally, the result of one subject quantitatively approximated the predicted value of the marginal value theorem, which was contradictory to previous studies in which the obtained values were higher than the predicted values. The present results support the validity of the marginal value theorem and suggest the importance of the evaluation index for foraging theories.

\section{$\mathrm{P}-25-19$}

\section{Japanese fire belly newts form CS-US association in appetitive classical conditioning} TOHRU TANIUCHI

School of Humanities, Kanazawa University

The present study examined associative mechanisms in the appetitive conditioning in newts. Newts were housed individually in an alley-like cage which had a middle dark place and bright areas in both ends. An object stimulus was presented as CS in one end and then a food US was presented in the opposite end. Reliable development of sign tracking responses but not goal tracking responses was observed. Subsequent US-devaluation tests revealed that the sign-tracking CR was reliably decreased by satiating newts. The results suggest that CSUS association but not CS-response association is mainly responsible for the development of $\mathrm{CR}$ in the present setting.

\section{$\mathrm{P}-25-20$}

Do sexually-unmatured juvenile crows discriminatively interact between males and females?

NANA TAKAHASHI ${ }^{1)}$, EI-ICHI IZAWA ${ }^{2)}$, TOSHIKAZU HASEGAWA ${ }^{1}$

${ }^{1)}$ Graduate School of Arts and Sciences, The University of Tokyo,

${ }^{2)}$ Department of Psychology, Keio University

Sexually different behavior has been extensively studied in birds under reproductive contexts. However, little is known whether unmatured juveniles show sexually different behavior. Large-billed crow (Corvus macrorynchos) juveniles form heterosexual groups during 3-year juvenile period. Such long-term juvenilehood allows investigating the sexual difference of juvenile behavior outside reproductive contexts. In this study, we examined whether 1.5 -year-old juvenile crows behaved discriminatively against unfamiliar males and females, who never met before, in a dyadic encounter paradigm. In this paradigm, two unfamiliar individuals were allowed to interact freely in an outdoor aviary for $5 \mathrm{~min} \times 3$ trials with 1-day inter-trial intervals. We tested 5 male-male dyads and 4 male-female dyads and confirmed the formation of clear dominance in 4 male-male dyads and 3 male-female dyads where one specific individual consistently showed submission to the other one across the 3 encounters. In terms of the number of displays and the latency to the first interaction, no difference of dominant 
males' aggressions to subordinates was found between unfamiliar males and females in their first encounters, nor of submissive behavior to the dominants between subordinate males and females. These results support a possibility that juvenile crows interact indiscriminatively between males and females.

\section{$\mathrm{P}-25-21$ \\ Do goldfishes perceive congruency of bright and dark between color and pitch? MAKOTO TAKAHASHI \\ Faculty of Letters, Otani University.}

Our human express high pitch as brighter sound, or low pitch as darker sound. The expressions imply that the auditory sense evoked visual sense. In this study, we examined whether goldfishes perceived commonality between brighter sound and light or darker sound and light. Ten goldfishes explored T-shape pathway for 5 minute. In the exploration, goldfishes were presented a sound which $1000 \mathrm{~Hz}$ and $100 \mathrm{~Hz}$ of pure tone was changed alternately every 30 seconds. Simultaneously, the fish presented two visual stimuli, congruent to the sound at left (right) side of the pathway or incongruent to the sound at the right (left) side of the pathway. For congruent visual stimulus, black color combined with $100 \mathrm{~Hz}$ and white color combined with $1000 \mathrm{~Hz}$. For the incongruent stimulus, the combination was reversed. Goldfishes experienced the exploration for 4 sessions, counterbalanced left-right position and the order of sound. I measured staying time for each visual stimulus as the preference. I presumed that if the fish perceived congruency between the sound and color, they should show preference to congruent or incongruent visual stimulus. As a result, goldfishes did not show any preference to visual stimulus. This result would suggest that fish did not have the perception.

\section{$\mathrm{P}-25-22$}

Acquired sexual preferences depending on skin colors of surrounding fish in medaka

Makiko Kamijo, Emi Ohya, Hiroka Shimada, Mayuka Ikawa, Shoji Fukamachi

Department of Chemical and Biological Sciences, Japan Women's University

We examined sexual preference of male medaka (Oryzias latipes). Mate-choice experiments were conducted between two skin-color variants, color interfere (ci) and Actb-SL $\alpha$ :GFP, which are away from each other by a single-gene expression. $c i$ is a mutant of the somatolactine alpha $(S L \alpha)$ gene exhibiting a pale gray color, whereas Actb-SL $\alpha$ :GFP is a transgenic ci that overexpresses $S L \alpha$ exhibiting causing a dark yellow color. These strains were reared with various tank mates from hatch until sexual maturation. Matured males were allowed to mate with novel two females of different strains for 30 minutes, and the number of courtship behaviors to each female was counted to assess his preference. Preference test with pair of two strains female was repeated for four successive days. When these strains had been reared separately, the males strongly preferred females of the same strain. However, if the strains had been reared in a mixed condition or each fish was reared individually, the males significantly increased interests in females of the other strain. These results indicate that sexual preferences of males are established being affected by fish they grow up with.

\section{$\mathrm{P}-25-23$}

A new multi-purpose operant test chamber for the studies of fish cognition

Ryosuke ChIYONOBU, KAZUO FujITA

Graduate school of Letters, Kyoto University

We made a new automated operant conditioning chamber with an infrared touch-sensitive LCD monitor used for the study of fish cognition. Fish typically spend their whole life underwater, and they are supposed to have evolved unique cognitive abilities to adapt to diverse of aquatic environments. Teleost fish have been tested for their learning and 
perception from late 1950's, in operant chambers, but studies of cognitive abilities have been rare because of procedural limitations. Our apparatus has three advantages. First, our use of the LCD monitor makes the systematic control of the stimulus presentation easy. Second, procedures traditionally used to test terrestrial animals can be comfortably adapted to fish because the apparatus works in almost exactly the same manner as traditional operant chambers. Third, this apparatus can be easily adapted to various kind of fish. These characteristics are helpful for comparative studies of cognition among diverse vertebrates and among different species of fish. Here we show how precisely the infrared touch screen detects objects in the water and how fish learned a simple association between positive reinforcements and a target stimulus. In addition, we will report the process of making an operant chamber filled with water.

\section{$\mathrm{P}-25-24$}

Concurrent acquisition of preference and aversion in place-odour compound conditioning with methamphetamine as the US

Yutaka Kosaki, SHigeru WatanaBe

Department of Psychology, Keio University

In a modified version of conditioned place preference (CPP) task, we studied in mice whether pairing methamphetamine US (METH; $2.0 \mathrm{mg} / \mathrm{kg}$, i.p.) with multimodal compound CS results in differential conditioning towards different elements of the compound CS. Each CS consisted of a conventional compartment cue (e.g. black walls and rod floor) and an object scented with essential oil. The compound CS+ was repeatedly paired with METH while another compound (CS-) was paired with saline. In elemental preference tests conducted after eight days of differential conditioning, mice showed a preference for the CS+ compartment over the CS- compartment, whereas they were indifferent to the CS+ object and the CSobject. After a total of 16 days of conditioning, however, mice developed a significant aversion towards the CS+ object while maintaining the preference for the CS+ compartment. Neither the degree of place preference nor object aversion differed between the group just described and the two control groups in which only one of the two stimulus elements was correlated with the presence of METH (Group Place and Group Object)- lack of reciprocal overshadowing. The results are discussed in relation to the conditioned opponent-process theory and the etiology of compulsive drug taking.

\section{$\mathrm{P}-25-25$}

Effects of emotional conditioning and propranolol on maze learning of rats

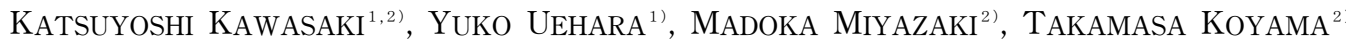

${ }^{11}$ Department of Psychology, Hoshi University,

${ }^{2)}$ Department of Psychology, Japan Women's University

The purpose of this experiment was to clarify the effects of emotional conditioning and propranolol (\&beta blocker) on maze learning of rats. Twenty Wistar rats were used as subjects. After 7 days of habituation to experimenters and the maze, half of subjects were emotionally conditioned with tone (CS) paired with electric shock given from the grid of floor in a shock box. The same tone was given to the other half of subjects but without shock. Six days of emotional conditioning was followed by 6 days of L/R-discrimination maze learning. All subjects were trained to run in the maze and choose either right or left goal box (individually fixed) for food pellets reward. CS (3sec.) was given just before the start door was opened in every trial. Propranolol $(10 \mathrm{mg} / \mathrm{kg})$ was administered to half of the respective groups $5 \mathrm{~min}$. before daily training on the day $1-3$. Saline was administered to the other half. No compound was administered to any animal on the day 4-6. The result showed that both emotional conditioning and propranolol enhanced maze performance of rats. This may suggest the possibility that two factors promote maze learning independently each. 
$\mathrm{P}-25-26$

Fear conditioning in physically restricted mice

KAZUYUKI YAMADA ${ }^{1)}$, KeISUKE OTA ${ }^{2)}$, MAYA OdAGAWA ${ }^{2)}$, TAKASHI MATSUMOTO ${ }^{2)}$, MASANORI MURAYAMA ${ }^{2)}$

${ }^{1)}$ School of Management, Shizuoka Sangyo University, ${ }^{2}$ BSI, RIKEN

Classical fear conditioning is widely used in neuroscience and behavioral psychology and as such, there are neural circuits believed implicated in this form of learning. However, activity at the level of the individual neuron during conditioning is not well-understood. Consequently, we developed a new experimental method for measuring cortical electrical activity in physically restrained mice as they underwent a conditioning treatment designed to associate a particular auditory tone with pain and elicit a reaction of fear. Each mouse's movement was restricted, its head and hind paws on the apparatus, with an electrode attached the left hind paw with adhesive tape. Then, the mouse was administered an electric stimulus in the left hind paw at the end of the tone presentation. The tone-stimulus pairing was repeated 30 times. Forty-eight hours after conditioning, a test trial was conducted in another laboratory. The test trial consisted of two parts; in the first half there was no tone and in the second half the tone was played. The mice showed significantly longer freezing in the second half than during the first half. Our results showed that mice quickly come to associate a tone with the threat of an electric stimulus in physically restricted settings.

\section{$\mathrm{P}-25-27$}

Do mice discriminate biological motions of mice?

TAKEShi ATSUMi ${ }^{1)}$, MASAKAZU IDE ${ }^{1,2)}$, MAKoto WADA ${ }^{1)}$

${ }^{11}$ Department of Rehabilitation for Brain Functions, Research Institute of National Rehabilitation Center for Persons with Disabilities,

${ }^{2)}$ JSPS Research Fellow

Recent studies using mice have shown better visual sensitivity for their social communication than previously thought. It is, however, still unclear what kind of visual information is critical. Here, we sought to elucidate their sensitivity to the bodily motion information in biological motion (BM) displays. We assessed the preference for BM display in mice (Mus musculus). The apparatus was a three-chambered box with opening between the chambers, and each side of the chamber had a monitor. We employed 4 wild-type male mice $(\mathrm{C} 57 \mathrm{BL} / 6)$ and recorded their walking by a digital video camera. We put point-lights on their remarkable body parts and created the movie with the white points on dark background (BM movies). For creating scramble motion (SM) movies, we randomly rearranged the original configuration of the white points. In testing, each subject introduced to the center, BM and SM version of the movies appeared at the peripheral two chambers. The subject explored within the apparatus for $10 \mathrm{~min}$. Results showed the mice spent more time in the chamber with SM displays. This indicates the scramble motion were relatively novel for the mice, and also suggests they could detect socially familiar cues from the biological motion displays.

\section{$\mathrm{P}-25-28$}

\section{Recollection of What-Where-Which memory in degus}

Toru Betsuyaku, Mana Tsuzuki, Kazuo Fujita

Graduate School of Letters, Kyoto University

Integration of what-where-when of one's personal experience is a key feature of episodic memory. However, there is argument that when is no more than an occasion setter of the event and functionally equivalent to which environmental context. In fact, rats have been reported to recall what, where, and which - object, place, and context - as an integrated memory (Eacott, et al., 2005). Here we report that degus do the same. In two E-shaped mazes with different contexts regarding wall and floor, degus were entered into the middle arm and 
first explored the maze with two trial-unique objects placed at each end of the backbone of the $\mathrm{E}$, with their location reversed between contexts. After being habituated to one of the objects in the holding cage, the degus re-explored the maze. Subjects explored the nonhabituated object in these baseline trials. In the test in which the objects were located at the end of the outer arms, degus chose the arm where they could expect the non-habituated object significantly more often than the other. As the objects were non-visible from subjects at choice, the result was not explainable by object familiarity derived from vision, but suggests recollection of an integrated what-where-which memory.

\section{$\mathrm{P}-25-29$}

Aging effects on activity, object memory, and spatial learning in Octodon degus

\section{TOMOKO UEKITA}

Department of Psychology, Kyoto Tachibana University

Octodon degus is considered an animal model of Alzheimer's disease because naturally occurring age-related brain pathology has been reported in these animals at 6 years of age. To assess age-related behavioral and cognitive alterations, we compared the locomotor activity, object memory, and spatial learning ability of aged (57 months old) with young ( 7 months old) degus. Aged degus traveled less horizontal distance than young degus did in an insulated chamber. For the object memory test, two objects were presented in the sample trial, and after delays of $3 \mathrm{~min}, 30 \mathrm{~min}$, or $3 \mathrm{~h}$, one of the two objects was replaced by a novel object in the test trial. Young degus showed longer exploration times for the novel object than for the sample object at all three delays, whereas aged degus showed similar exploration times for the objects at delays of $30 \mathrm{~min}$ and $3 \mathrm{~h}$. For the T-maze spatial task, the performance of aged degus was the same as that for young degus in the test trial, with the starting arm changed to the opposite position. These results suggested that aging affected locomotor activity and object memory for delays greater than $30 \mathrm{~min}$ but not spatial learning in degus.

\section{$\mathrm{P}-25-30$}

Numerical discrimination of visual stimuli in miniature pigs RYOUSEI UENO ${ }^{1)}$, HIROKAZU INABA ${ }^{2)}$, TOHRU TANIUCHI ${ }^{3)}$

${ }^{1)}$ Farm of Ishikawa Prefectural University, ${ }^{2}$ Ishikawa Prefectural University,

${ }^{3)}$ Graduate School of Socio-Environmental Studies, Kanazawa University

We examined conditional place discriminations of numerical visual moving stimuli in two miniature pigs. One or two blue dots moving toward random directions were presented in a LCD. The dots of two-dot stimulus were $6 \mathrm{~cm}$ in diameter. The dot of one-dot stimulus was 2 $\mathrm{cm}$ in diameter in the first stage and the diameter gradually increased in later stages. There were response frames on both sides of the LCD. Nose poke responses to the right frame for one-dot stimulus, and to the left frame for two-dot stimulus, were reinforced for pig A. The responses to the opposite side frame were reinforced for Pig B. Both pigs achieved high correct performance when the dot of one-dot stimulus was $2 \mathrm{~cm}, 3 \mathrm{~cm}$ or $4 \mathrm{~cm}$ in diameter. But, the correct rates decreased when the dot was more than $4 \mathrm{~cm}$ in diameter. Then the least distance between the two dots of two-dot stimulus were changed from $1.4 \mathrm{~cm}$ to $6 \mathrm{~cm}$. Although Pig A achieved high correct rates when the dot of one-dot stimulus was $6 \mathrm{~cm}, 8.5$ $\mathrm{cm}$ or $12 \mathrm{~cm}$ in diameter, performance of Pig B did not improve.

\section{$\mathrm{P}-25-31$}

Self-scratching contagion in free-ranging Japanese macaques (Macaca fuscata)

\section{MASATAKA UENO}

Graduate School of Human Sciences, Osaka University

Emotional contagion is considered to be linked to social bonds and empathy in animal societies. Self-scratching is the expression of anxiety or distress, yet this behavior has received 
little attention in literature on emotional contagion. We investigated whether observing selfscratching behavior elicited the same behavior from the observer and whether affiliative relationships (i.e., social bonds) facilitate scratching contagion in free-ranging Japanese macaques. We used a focal-animal sampling method, targeting 17 females at Katsuyama, Okayama Prefecture, Japan. We evaluated affiliative relationships, which were defined by standard proximity rates. As a result, female Japanese macaques scratched themselves at a higher rate within three minutes when they observed other females scratching themselves than when they did not. In addition, self-scratching behavior occurred sooner in the observe condition than in the unobserve condition, and the differences between the two conditions were larger in affiliated pairs than in unaffiliated pairs. These results indicate that selfscratching contagion occurs between adult females in free-ranging Japanese macaques and that affiliative relationships positively affect such self-scratching behavior.

\section{$\mathrm{P}-25-32$}

Order representations shared cognitive resources with spatial working memory in apes IKUMA ADACHI ${ }^{1}$, RACHEL DIAMOND ${ }^{2)}$, ROBERT HAMPTON ${ }^{2)}$

${ }^{1)}$ Primate Research Institute, Kyoto University,

${ }^{2)}$ Yerkes National Primate Research Center, Emory University

Humans organize their thinking about order and quantities using mental representations with spatial characteristics. Many nonhuman species also represent space, quantity, and order and so might show similar space-order interactions in nonhuman primates. There is limited evidence suggesting that similar spatial representations support memory for order in nonhuman primates. We directly tested whether the ability to order lists in apes is dependent on a spatial representation mechanism. Subjects were trained to remember the spatial location of a stimulus on a touchscreen computer. After subjects learned the spatial memory task, we embed either an order task or a control task into the delay of the spatial memory task. If a spatial representation supports memory for order in nonhuman primates then performance on the order task would be impaired when it is embedded within the spatial memory task and spatial memory resources are limited. In contrast the spatial hypothesis does not predict a deficit in performance on a non-spatial task. Therefore, a non-spatial matching-to-sample test was employed as a control task. The obtained results support our hypothesis that the ability to order lists share cognitive resources with spatial memory. 